\title{
Article
}

\section{The JCMT and Herschel Gould Belt Surveys: a comparison of SCUBA-2 and Herschel data of dense cores in the Taurus dark cloud L1495}

Ward-Thompson, D, Pattle, K, Kirk, Jason Matthew, Marsh, K, Buckle, J, Hatchell, J, Nutter, DJ, Griffin, MJ, Di Francesco, J, André, P and, et al.

Available at http://clok.uclan.ac.uk/15790/

Ward-Thompson, D ORCID: 0000-0003-1140-2761, Pattle, K, Kirk, Jason Matthew ORCID: 0000-0002-4552-7477, Marsh, K, Buckle, J, Hatchell, J, Nutter, DJ, Griffin, MJ, Di Francesco, J et al (2016) The JCMT and Herschel Gould Belt Surveys: a comparison of SCUBA-2 and Herschel data of dense cores in the Taurus dark cloud L1495. Monthly Notices of the Royal Astronomical Society, 463 (1). pp. 1008-1025. ISSN 0035-8711

It is advisable to refer to the publisher's version if you intend to cite from the work. http://dx.doi.org/10.1093/mnras/stw1978

For more information about UCLan's research in this area go to http://www.uclan.ac.uk/researchgroups/ and search for <name of research Group>.

For information about Research generally at UCLan please go to http://www.uclan.ac.uk/research/

All outputs in CLoK are protected by Intellectual Property Rights law, including Copyright law. Copyright, IPR and Moral Rights for the works on this site are retained by the individual authors and/or other copyright owners. Terms and conditions for use of this material are defined in the policies page. 


\title{
The JCMT and Herschel Gould Belt Surveys: a comparison of SCUBA-2 and Herschel data of dense cores in the Taurus dark cloud L1495
}

\author{
D. Ward-Thompson, ${ }^{1 \star}$ K. Pattle,${ }^{1}$ J. M. Kirk, ${ }^{1}$ K. Marsh, ${ }^{2}$ J. Buckle,${ }^{3,4}$ J. Hatchell, ${ }^{5}$ \\ D. J. Nutter, ${ }^{2}$ M. J. Griffin, ${ }^{2}$ J. Di Francesco, ${ }^{6,7}$ P. André, ${ }^{8}$ S. Beaulieu, ${ }^{9}$ D. Berry, ${ }^{10}$ \\ H. Broekhoven-Fiene, ${ }^{7}$ M. Currie, ${ }^{10}$ M. Fich, ${ }^{9}$ T. Jenness, ${ }^{11}$ D. Johnstone, ${ }^{6,7}$ \\ H. Kirk, ${ }^{6}$ J. Mottram, ${ }^{12,13}$ J. Pineda, ${ }^{14}$ C. Quinn, ${ }^{2}$ S. Sadavoy, ${ }^{13}$ \\ C. Salji, ${ }^{3,4}$ S. Tisi, ${ }^{9}$ S. Walker-Smith, ${ }^{3,4}$ G. White, ${ }^{15,16}$ T. Hill, ${ }^{17}$ \\ V. Könyves, ${ }^{8}$ P. Palmeirim ${ }^{8}$ and S. Pezzuto ${ }^{18}$
}

Affiliations are listed at the end of the paper

Accepted 2016 August 5. Received 2016 August 4; in original form 2016 March 23

\begin{abstract}
We present a comparison of Submillimetre Common User Bolometer Array-2 (SCUBA-2) 850- $\mu \mathrm{m}$ and Herschel 70-500- $\mu \mathrm{m}$ observations of the L1495 filament in the Taurus Molecular Cloud with the goal of characterizing the SCUBA-2 Gould Belt Survey (GBS) data set. We identify and characterize starless cores in three data sets: SCUBA-2 $850-\mu \mathrm{m}$, Herschel 250- $\mu \mathrm{m}$, and Herschel $250-\mu \mathrm{m}$ spatially filtered to mimic the SCUBA-2 data. SCUBA-2 detects only the highest-surface-brightness sources, principally detecting protostellar sources and starless cores embedded in filaments, while Herschel is sensitive to most of the cloud structure, including extended low-surface-brightness emission. Herschel detects considerably more sources than SCUBA-2 even after spatial filtering. We investigate which properties of a starless core detected by Herschel determine its detectability by SCUBA-2, and find that they are the core's temperature and column density (for given dust properties). For similartemperature cores, such as those seen in L1495, the surface brightnesses of the cores are determined by their column densities, with the highest-column-density cores being detected by SCUBA-2. For roughly spherical geometries, column density corresponds to volume density, and so SCUBA-2 selects the densest cores from a population at a given temperature. This selection effect, which we quantify as a function of distance, makes SCUBA-2 ideal for identifying those cores in Herschel catalogues that are closest to forming stars. Our results can now be used by anyone wishing to use the SCUBA-2 GBS data set.
\end{abstract}

Key words: stars: formation - ISM: clouds - ISM: Individual objects: Taurus and L1495.

\section{INTRODUCTION}

Stars form in dense cores within molecular clouds (e.g. Strom, Strom \& Grasdalen 1975; Wilking, Lada \& Young 1989; WardThompson et al. 1989; Ballesteros-Paredes, Klessen \& VázquezSemadeni 2003). Recent work has indicated that cores form preferentially in filaments within molecular clouds (e.g. André et al. 2010, 2014; Molinari et al. 2010). Those cores that do not contain proto-stars are known generically as starless cores (Beichman et al. 1986). When starless cores become dense enough to be gravitationally bound, they are known as pre-stellar cores (Ward-Thompson

*E-mail: dward-thompson@uclan.ac.uk et al. 1994; Di Francesco et al. 2007; Ward-Thompson et al. 2007a). They then collapse to form Class 0 (André, Ward-Thompson \& Barsony 1993) and then Class I (Lada 1987; Wilking et al. 1989) protostars, which can be seen as point-like sources in the near- and mid-infrared (e.g. André, Ward-Thompson \& Barsony 2000).

To trace the structure within molecular clouds, and particularly the filamentary structure, it is necessary to use the far-infrared (e.g. André et al. 2010). The ESA Herschel Space Observatory ${ }^{1}$ (Pilbratt et al. 2010) has been especially successful in this regard

\footnotetext{
${ }^{1}$ Herschel is an ESA space observatory with science instruments provided by European-led Principal Investigator consortia and with participation from NASA.
} 
(e.g. André et al. 2010; Molinari et al. 2010). In particular, the large collecting area and powerful science payload of Herschel allow one to perform high-resolution, sensitive, mid- and far-infrared imaging photometry using the PACS (Poglitsch et al. 2010) and SPIRE (Griffin et al. 2010) instruments - often simultaneously (e.g. André et al. 2010).

However, Herschel is so sensitive to molecular cloud structure - particularly SPIRE - that it sees all of the cores within a cloud (e.g. Men'shchikov et al. 2010). It detects many starless cores that are not pre-stellar in nature (e.g. Ward-Thompson et al. 2010), as well as those that are (e.g. André et al. 2010). Consequently, if one is to confirm which cores are gravitationally bound (pre-stellar), and hence destined to form stars, additional information is needed. The minimum information required in order to determine the virial balance of a starless core is a measure of the core's mass and size (obtainable from submillimetre continuum observations) and internal velocity dispersion (requiring measurement of the core's internal linewidth). In order to accurately determine both the virial boundedness of the core and the mechanism by which it is confined (i.e. by self-gravity or by external pressure), measures of the external pressure on and magnetic field strength within the core are also required (cf. Pattle et al. 2015).

Submillimetre continuum mapping identified the first genuine pre-stellar cores (Ward-Thompson et al. 1994). Therefore, in this paper we test the hypothesis that submillimetre continuum mapping with the Submillimetre Common User Bolometer Array 2 (SCUBA-2) camera can be used to discriminate between those cores detected by Herschel which are most likely to be gravitationally bound (pre-stellar), and those which are unbound and transient in nature. In the absence of the spectroscopic data required to perform a virial analysis, the stability of starless cores is often assessed using density-based criteria such as the Jeans criterion (Jeans 1928) or the Bonnor-Ebert criterion (Ebert 1955; Bonnor 1956). In these analyses, denser cores are, for a given temperature, more likely to be gravitationally bound.

The presence of the atmosphere means that ground-based submillimetre continuum mapping instruments must inevitably be subject to limitations on absolute sensitivity and large-scale structure recovery to which space-based instruments are not (e.g. Sadavoy et al. 2013). However, the selection effects introduced by these constraints may result in a ground-based detection, or otherwise, of a core detected using space-based instrumentation providing additional information about the properties of that core than the space-based data alone could provide. In this paper we identify and quantify the selection effects determining the detection, or otherwise, with SCUBA-2 of a starless core detected with Herschel. We further investigate whether these selection effects allow the identification of the densest cores in the Herschel data through their detection, or otherwise, by SCUBA-2.

The L1495 region of Taurus appears as an obscuring dark cloud on optical images (Barnard 1907; Lynds 1962), with a linear, or filamentary, structure, coming to a head at a small globule that is referred to as L1495A (Benson \& Myers 1989; Lee, Myers \& Tafalla 2001). Hacar et al. (2013) studied the L1495 filament and found it to be consistent with the filamentary star formation model (André et al. 2014) favoured by Herschel observations of star-forming regions (André et al. 2010). Lee et al. (2001) found evidence for asymmetric line profiles in the southern part of L1495A, apparently indicating collapse or contraction. However, this is close to the Herbig Ae/Be star, V892 Tau, which is clearly affecting at least the southern part of L1495A, so interpreting asymmetric line profiles is made more complex in this area. The Herschel data of the L1495 filament
(Marsh et al. 2016) and follow-up ammonia data from the Green Bank Telescope (Seo et al. 2015) identified a number of pre-stellar cores in the L1495 filament.

The SCUBA-2 observations presented here were carried out as part of the Gould Belt Legacy Survey (GBLS) on the James Clerk Maxwell Telescope (JCMT) in Hawaii (Ward-Thompson et al. 2007b). The full SCUBA-2 data were presented by Buckle et al. (2015). The Herschel observations presented in this paper were carried out as part of the Herschel Gould Belt Survey (HGBS) guaranteed-time key programme (André et al. 2010). The full Herschel data were presented by Marsh et al. (2014, 2016). Herschel observations of the southern part of the L1495 filament were presented by Palmeirim et al. (2013). In this paper we present a comparison between the two sets of data (see Figs 1 and 2). We use the Herschel data (Marsh et al. 2016) as a comparison data set to determine which sources are detected by SCUBA-2, and what properties are important for a SCUBA-2 detection.

\section{OBSERVATIONS}

\subsection{SCUBA-2}

The SCUBA-2 (Holland et al. 2013) observations used here form part of the JCMT Gould Belt Legacy Survey (GBLS; WardThompson et al. 2007b). The L1495 region of the Taurus molecular cloud was observed with the SCUBA-2 camera in 22 observations taken between 2011 October and 2013 July. Continuum observations at 450 and $850 \mu \mathrm{m}$ were made using fully sampled 15-, 30, and 60-arcmin diameter circular regions (PONG900, 1800 and 3600 mapping modes; Bintley et al. 2014). Larger regions were mosaicked with overlapping scans. The final output map is centred at a position of R.A. (2000) $04^{\mathrm{h}} 17^{\mathrm{m}} 54^{\mathrm{s}}$, Dec. $(2000)+28^{\circ} 05^{\prime} 24^{\prime \prime}$. These data were presented by Buckle et al. (2015), in which full details of the observations are given. Here, we briefly reiterate the key points. We only use the $850-\mu \mathrm{m}$ data. ${ }^{2}$

The data were reduced as part of the Internal Release 1 data set, using an iterative map-making technique (MAKEMAP in SMURF, Chapin et al. 2013), and gridded to 6 -arcsec pixels at $850 \mu \mathrm{m}$. The iterations were halted when the map pixels, on average, changed by $<0.1$ per cent of the estimated map rms. The initial reductions of each individual scan were co-added to form a mosaic from which a signal-to-noise ratio mask was produced for each region. The final mosaic was produced from a second reduction using this mask to define areas of emission. Detection of emission structure and calibration accuracy are uncertain outside of the masked regions. The mask used in the reduction can be seen in the quality array in the reduced datafile (see Buckle et al. 2015).

A spatial filter of 10 arcmin was used in the reduction, which means that flux recovery is robust for sources with a Gaussian full width at half-maximum (FWHM) less than 2.5 arcmin. Sources between 2.5 and 7.5 arcmin may be detected, but both the flux density and the size will be underestimated because Fourier components with scales greater than 5 arcmin are essentially removed by this filtering process. Detection of sources larger than 7.5 arcmin is dependent on the mask used for each reduction.

The data were calibrated in Jy/pixel, using an aperture flux conversion factor (FCF) of $2.34 \mathrm{Jy} / \mathrm{pW} / \operatorname{arcsec}^{2}$ at $850 \mu \mathrm{m}$, derived from

\footnotetext{
${ }^{2}$ The SCUBA-2 data used in this paper are available at: http://dx.doi. org/10.11570/16.0002 (Buckle et al. 2015).
} 


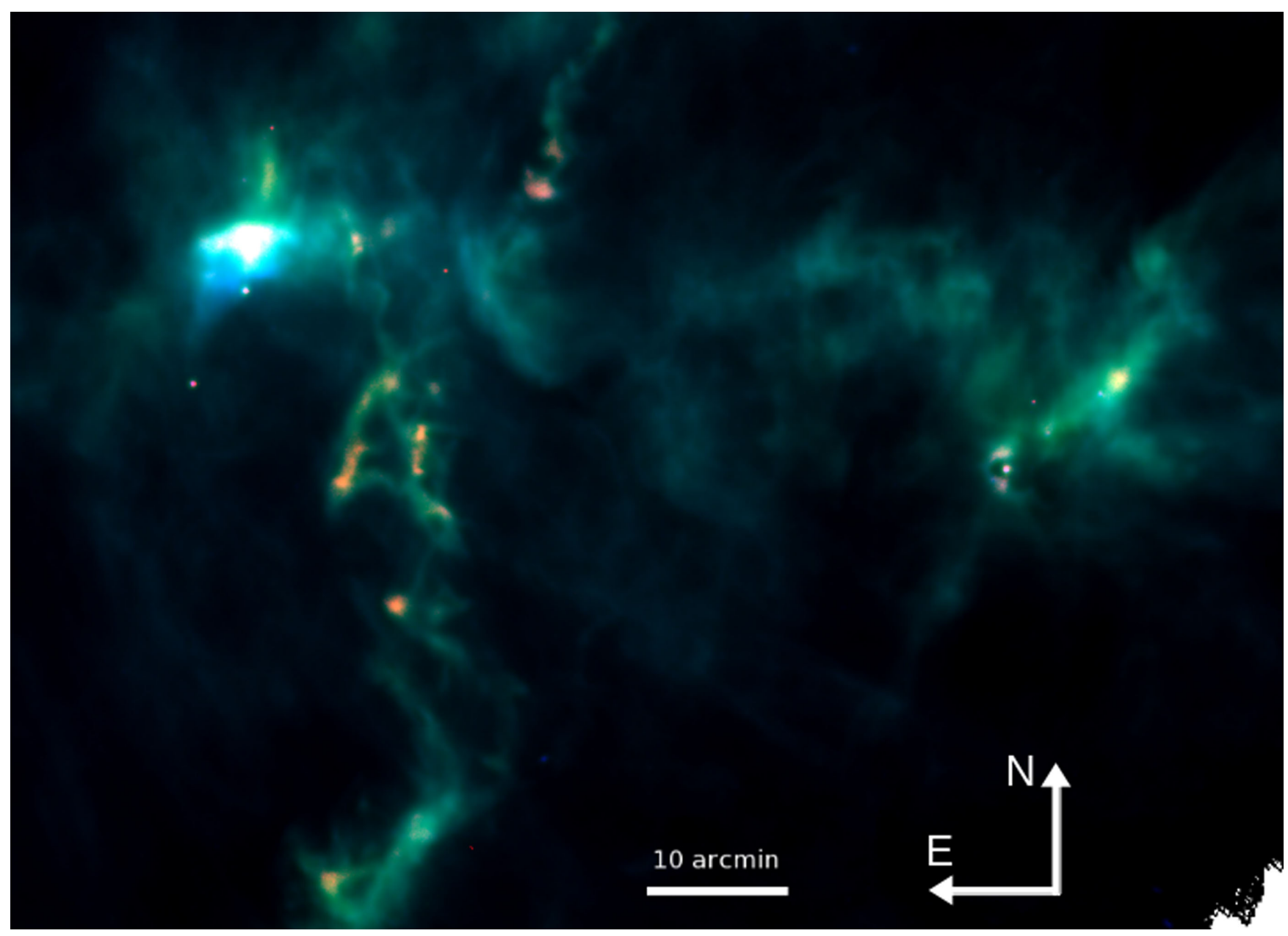

Figure 1. The L1495 region, as observed using SCUBA-2 and Herschel. Red channel: SCUBA-2 850- $\mu \mathrm{m}$ emission. Green channel: SPIRE 500- $\mu \mathrm{m}$ emission. Blue channel: SPIRE 250- $\mu \mathrm{m}$ emission. Note how most of the structure of the cloud is detected by Herschel and is seen as blue-green emission in this image. However, only some of the sources are picked out by SCUBA-2, and show up as red in this image.

average values of JCMT calibrators (Dempsey et al. 2013), and correcting for the pixel area. The PONG scan pattern leads to lower noise in the map centre and overlap regions, while data reduction and emission artefacts can lead to small variations in the noise over the whole map.

\subsection{Herschel Space Observatory}

The Herschel Space Observatory is a 3.5-m-diameter telescope, which operates in the far-infrared and submillimetre regimes (Pilbratt et al. 2010). The comparison Herschel data used in this paper were taken as part of the HGBS (André et al. 2010) and were presented by Marsh et al. (2016). They were taken simultaneously with the Photodetector Array Camera and Spectrometer, PACS (Poglitsch et al. 2010), and the Spectral and Photometric Imaging Receiver, SPIRE (Griffin et al. 2010; Swinyard et al. 2010), using the combined fast-scanning (60 arcsec/s) SPIRE/PACS parallel mode. See André et al. (2010) and Marsh et al. (2016) for details of the observations and the data reduction process.

\section{RESULTS}

As SCUBA-2 is a ground-based instrument, while Herschel is space-based, the ability of SCUBA-2 to recover submillimetre emission is restricted in comparison to Herschel. Although the data sets used in this work are of similar sensitivity (see below), the SCUBA2 data are affected by selection effects due to atmospheric emission and variability, to which the Herschel data are not. The result of this is that the emission detected by SCUBA-2 is a subset of that detected by Herschel. We here investigate what distinguishes those sources detected in both SCUBA-2 850- $\mu$ m and Herschel-SPIRE $250-\mu \mathrm{m}$ emission from those detected in $250-\mu \mathrm{m}$ emission alone. We restrict our analysis to extended, starless sources, in order to be able to accurately characterize our sources using only data at wavelengths $>100 \mu \mathrm{m}$ and we use a modified blackbody emission model.

The requirement for a SCUBA-2 detection of any source is a peaked $850-\mu \mathrm{m}$ surface brightness, as SCUBA-2 loses sensitivity to flux on larger spatial scales (see above). Thus, we hypothesize that the likely requirements for a SCUBA-2 detection of a starless core are for the core to be at high density (thus having a high surface brightness), low temperature (i.e. having a high ratio of long-wavelength to short-wavelength flux), and compactness (i.e. being small enough not to lose emission to the SCUBA-2 spatial filtering). These properties are related to one another: for starless cores of the same mass, in the absence of local heating, a dense core is expected to be colder than a rarefied core; and, trivially, a compact core will be denser than an extended core. The aim of this study is to distinguish which, if any, of these properties is of most importance 


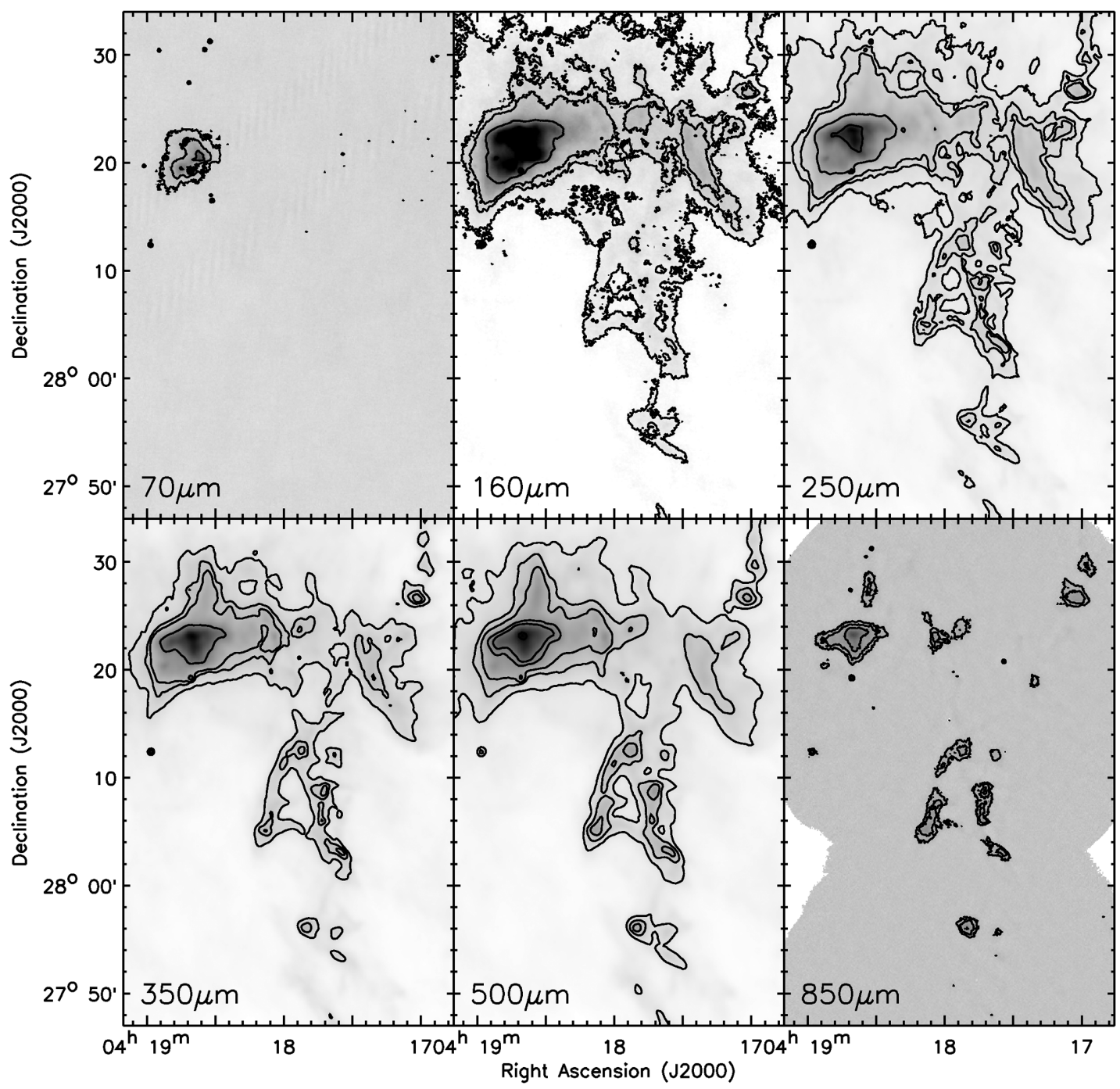

Figure 2. The centre of the L1495 region, as observed at the five Herschel wavelengths, 70, 160, 250, 350 and $500 \mu \mathrm{m}$ (Marsh et al. 2014, 2016) and SCUBA-2 $850 \mu \mathrm{m}$ (Buckle et al. 2015). Note how only some of the sources and structures seen at other wavelengths are detected by SCUBA-2 (lower right). Contour levels - $70 \mu \mathrm{m}$ : 0.01, 0.02, 0.05, $0.5 \mathrm{Jy} / 6$-arcsec pixel; $160 \mu \mathrm{m}: 0.05,0.08,0.1,0.2,0.5,1.0 \mathrm{Jy} / 6$-arcsec pixel; $250 \mu \mathrm{m}:$ as $160 \mu \mathrm{m} ; 500 \mu \mathrm{m}: 0.02,0.035,0.05$, $0.08,0.1,0.2,0.5 \mathrm{Jy} / 6-\operatorname{arcsec}$ pixel; $850 \mu \mathrm{m}: 0.005,0.01,0.02 \mathrm{Jy} / 6$-arcsec pixel.

in determining whether a starless core identified in Herschel data will also be detectable in SCUBA-2 850- $\mu \mathrm{m}$ emission.

Fig. 1 shows a three-colour image of the region mapped with SCUBA-2, in which $850 \mu \mathrm{m}$ is shown in red, superposed on the same region from the Herschel data, where $500 \mu \mathrm{m}$ is shown in green and $250 \mu \mathrm{m}$ is shown in blue. A number of cores and filaments can be seen. Herschel detects most of the cloud structure, seen as blue-green emission on this image, including many filaments, as previously seen in other regions. We also see many cores along the filaments, consistent with the recently proposed hypothesis that core formation on filaments is the dominant mode of star formation (André et al. 2014). However, only some of the sources are picked out by SCUBA-2, and show up as red in this image.

The brightest source in Fig. 1 is L1495A. Fig. 2 shows an enlargement of the L1495A region at each of the six wavelengths five from Herschel and one from SCUBA-2. It can be seen that only some of the sources and structures seen at other wavelengths are detected by SCUBA-2. Figs 4(a) and (b) show the full area covered at high signal-to-noise ratio by SCUBA- 2 at $850 \mu \mathrm{m}$.
L1495A can be seen clearly at all wavelengths in Figs 1 and 2, including with SCUBA-2 at $850 \mu \mathrm{m}$. In fact, the brightest peak coincides with the southern part of L1495A, namely L1495AS (Benson \& Myers 1989). The northern extension of L1495A, which is very much fainter, is L1495A-N (Lee et al. 2001). In the three-colour images in Fig. 1, a colour gradient can be seen from south to north across L1495A, from blue to green. This would tend to indicate a temperature gradient across this core, with the hotter material in the south, consistent with these earlier findings.

There is in fact a bright star, slightly further to the south of L1495A-S, which is V892 Tau (IRAS 04155+2812). This has a point source flux density in the IRAS Catalogue of $30 \mathrm{Jy}$ at $12 \mu \mathrm{m}$ and $100 \mathrm{Jy}$ at $25 \mu \mathrm{m}$, which declines slightly to $70 \mathrm{Jy}$ at $60 \mu \mathrm{m}$, before climbing again to $170 \mathrm{Jy}$ at $100 \mu \mathrm{m}$. The $100-\mu \mathrm{m}$ flux density almost certainly includes a contribution from L1495A-S, but otherwise, this is a Herbig Ae/Be star, and it is clearly heating L1495A-S, which is otherwise starless. A similar pattern of an externally heated core was modelled in Cepheus by Nutter, Stamatellos \& Ward-Thompson 


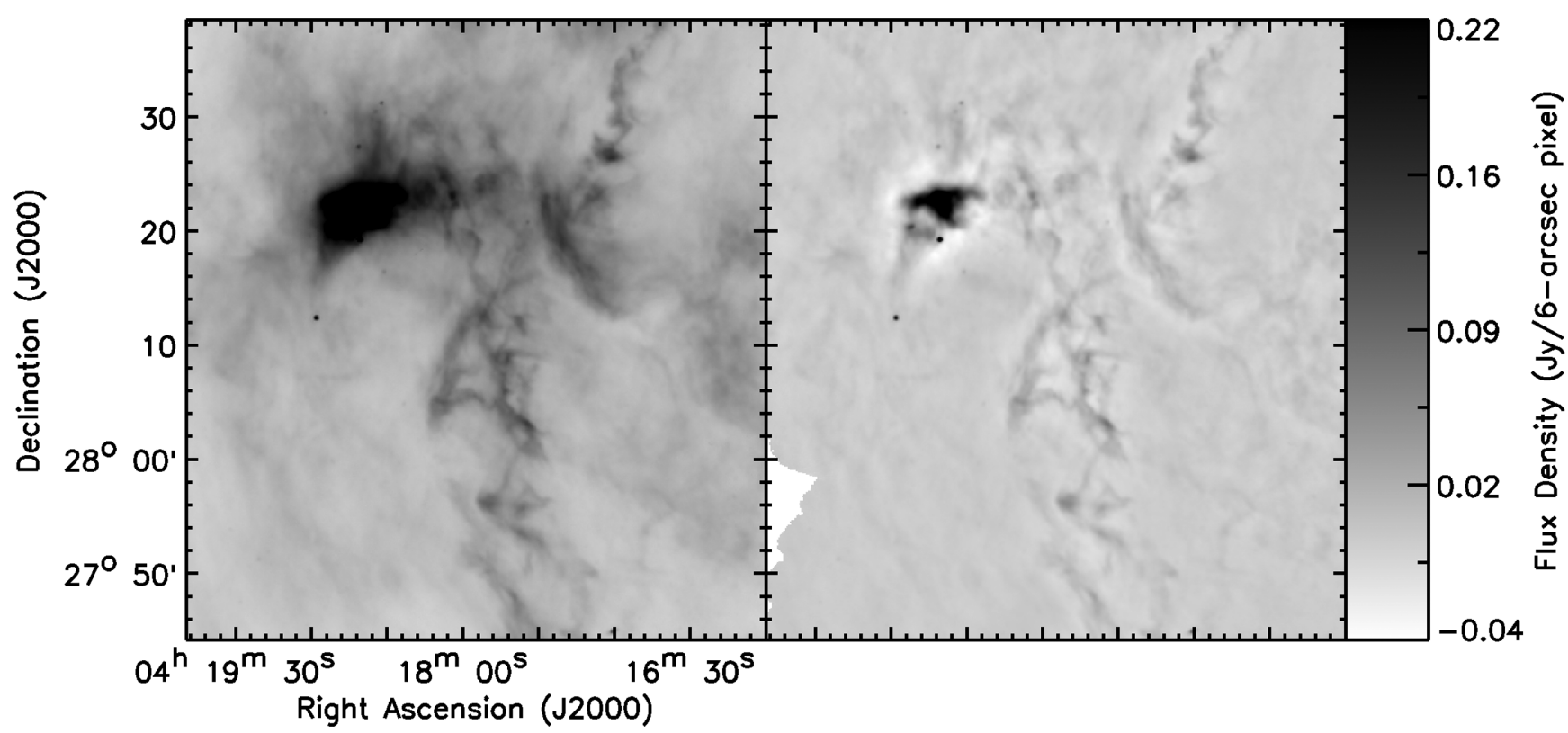

Figure 3. A comparison of the unfiltered (left) and filtered (right) SPIRE 250- $\mu$ m maps of the centre of the L1495 region. Note the loss of large-scale structure between the unfiltered and the filtered maps.

(2009), based on a combination of SCUBA and Akari data, and in that paper we cautioned that temperature gradients could affect the appearance of cores at long wavelengths such as these.

\section{COMPARISON OF SCUBA-2 DATA WITH HERSCHEL DATA}

In order to make SCUBA-2 and Herschel observations comparable, the large-scale structure must be removed from, and the SCUBA-2 mask must be applied to, the Herschel observations. To this end, the Herschel observations were processed through the SCUBA-2 pipeline, following the method described by Sadavoy et al. (2013) and Pattle et al. (2015). The Herschel data are scaled such that they represent a small perturbation on the SCUBA-2 850- $\mu$ m flux densities, and are added to the SCUBA-2 bolometer time series. The data reduction process, as described above, is repeated. The original SCUBA-2 reduction of the data is then subtracted from the Herschel + SCUBA-2 map, and the scaling applied to the Herschel data is reversed. This process produces a spatially filtered and masked version of the original Herschel observations, suitable for comparison with the SCUBA-2 data. This process is repeated once for each SCUBA-2 observing position, for which there were corresponding Herschel data, and the resulting filtered maps combined to form a mosaic.

The effect of the spatial filtering process on the SPIRE $250-\mu \mathrm{m}$ data set is shown in Fig. 3. Fig. 3 shows the loss of large-scale structure caused by the filtering process.

Three sets of sources were identified: sources in the $850-\mu \mathrm{m}$ SCUBA-2 data (hereafter referred to as SCUBA-2 sources), sources in the $250-\mu \mathrm{m}$ Herschel-SPIRE data (hereafter referred to as Herschel sources) and sources in the spatially filtered $250-\mu \mathrm{m}$ data (hereafter referred to as filtered-Herschel sources). Sources were identified using the source-finding algorithm CSAR (Kirk et al. 2013). CSAR is a dendrogram-based source-finding algorithm, which was run in its non-hierarchical mode on each of the three data sets. The criteria chosen for a robustly detected source were a peak flux density $F_{v}^{\text {peak }} \geq 4 \sigma$ and a minimum of a $3 \sigma$ drop in flux density between adjacent sources, where $\sigma$ is the rms noise level of the data (see Kirk et al. 2013). These stringent criteria were chosen in order to ensure that the sources we identified could be wellcharacterized, so that accurate comparisons could be made between cores detected in the different data sets.

We measured the $1 \sigma \mathrm{rms}$ noise on the low-variance regions of the SCUBA-2 $850-\mu \mathrm{m}$ map to be $0.9 \pm 0.2 \mathrm{mJy} / 6$-arcsec pixel and on the unfiltered $250-\mu \mathrm{m}$ map to be $1.0 \pm 0.2 \mathrm{mJy} / 6$-arcsec pixel. Measuring the noise on the filtered $250-\mu \mathrm{m}$ map produced values in the range 1.1-1.6 mJy/6-arcsec pixel. We adopted a value of $1.4 \pm 0.2 \mathrm{mJy} / 6$-arcsec pixel, as being representative. The increase in rms noise level in the filtered $250-\mu \mathrm{m}$ map over the unfiltered $250-\mu \mathrm{m}$ map is likely to be a result of the additional processing performed on the filtered map.

The regions of the SCUBA-2 map upon which source extraction was performed were those where the variance, as measured in the variance array, was $\leq 2(\mathrm{mJy} / 6 \text {-arcsec pixel })^{2}$. These are the two large regions marked in Figs 4(a) and (b). Noise levels across the Herschel maps are more uniform than those across the SCUBA-2 map. However, all sources detected in the Herschel data which were not fully located within one of the low-variance SCUBA-2 regions were excluded from further analysis, in order to allow an accurate comparison of the sources detected by the two instruments.

We identified 26 sources in the $850-\mu \mathrm{m}$ map, 211 sources in the $250-\mu \mathrm{m}$ map and 140 sources in the filtered $250-\mu \mathrm{m}$ map. Sources smaller than the beam were rejected in the source extraction process. We examined the source samples to determine whether any sources contained protostars based on whether they contained point sources at $70 \mu \mathrm{m}$ or shorter wavelengths. One protostellar source was identified in the SCUBA-2 sample, along with three in the 250$\mu \mathrm{m}$ sample and two in the filtered $250-\mu \mathrm{m}$ sample. These sources were excluded from further analysis, leaving us with 25 sources in the 850- $\mu \mathrm{m}$ map (shown in Figs 4a and b), 208 sources in the unfiltered $250-\mu \mathrm{m}$ map (shown in Figs A1a and b in the online appendix), and 138 sources in the filtered $250-\mu \mathrm{m}$ map (shown in Figs A2a and b in the online appendix). The 25 SCUBA-2 sources (named S1-S25) are listed in Table 1, while the Herschel sources 

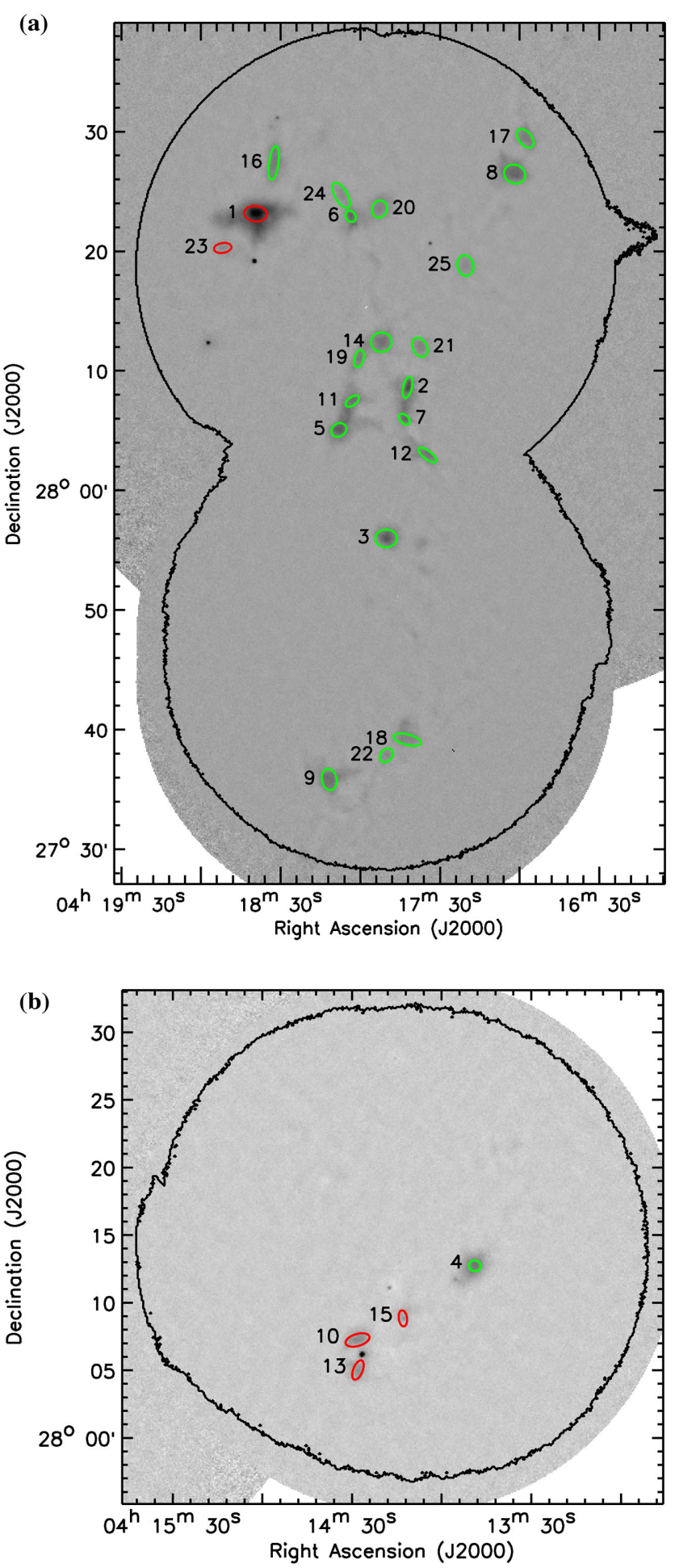

Figure 4. (a) Grey-scale image of the head of the L1495 filament, as mapped by SCUBA-2 at $850 \mu \mathrm{m}$. Sources detected in emission by SCUBA-2 are marked by small ellipses. Cores marked in red show signs of local heating (see text for details). The large-scale contour surrounds the region of lowest variance (cf. Buckle et al. 2015). Cores are numbered as in Table 1. (b) Same as Fig. 4(a), for the L1495 West region.
(H1-H208) and filtered Herschel sources (F1-F138) are presented along with the reduced SCUBA-2 data at the DOI listed in Section 2. For each SCUBA-2 source, Table 1 lists the name, right ascension and declination, measured major and minor FWHM sizes, position angle, flux densities as measured in filtered Herschel $160 \mu \mathrm{m}, 250$ $\mu \mathrm{m}, 350 \mu \mathrm{m}$ and $500 \mu \mathrm{m}$ and SCUBA-2 $850 \mu \mathrm{m}$ emission, and the equivalent sources in the Herschel and filtered-Herschel catalogues. The details of the Herschel tables are given at the same DOI.

We derived temperatures and masses for each of our sources using the spectral energy distribution (SED) measured from the Herschel and, in the case of the SCUBA-2 sources, the SCUBA-2 continuum data. The flux densities of the SCUBA-2 sources were measured across five wavebands (filtered $160-\mu \mathrm{m}$, filtered $250-\mu \mathrm{m}$, filtered $350-\mu \mathrm{m}$, filtered $500-\mu \mathrm{m}$ and $850-\mu \mathrm{m}$ ), all convolved to the SPIRE 500- $\mu \mathrm{m}$ resolution of 36 arcsec. The flux densities of the Herschel sources were measured across the unfiltered $160-\mu \mathrm{m}$, $250-\mu \mathrm{m}, 350-\mu \mathrm{m}$ and $500-\mu \mathrm{m}$ wavebands, while the flux densities of the filtered-Herschel sources were measured across the filtered $160-\mu \mathrm{m}, 250-\mu \mathrm{m}, 350-\mu \mathrm{m}$ and $500-\mu \mathrm{m}$ wavebands, in both cases also at 36-arcsec resolution. We convolved the maps to a common 36-arcsec resolution using the Herschel $\rightarrow$ Herschel and SCUBA$2 \rightarrow$ Herschel convolution kernels described by Pattle et al. (2015). Flux densities for each source were measured using elliptical apertures with major and minor axes of twice the major and minor FWHM returned by CSAR. This would enclose 99.5 per cent of the flux in a Gaussian distribution.

Note that the $850-\mu \mathrm{m}$ flux densities listed in Table 1 do not have the SCUBA-2 aperture photometry corrections discussed by Dempsey et al. (2013) applied to them. The SCUBA-2 aperture photometry corrections are determined for point sources, and account for flux in the secondary beam of the JCMT not enclosed by a small aperture (the JCMT's secondary beam has a FWHM of 48 arcsec at $850 \mu \mathrm{m}$; see Dempsey et al. 2013). We do not use these aperture photometry corrections in this work, as their applicability to either extended sources or non-circular apertures is not certain. As noted above, the major and minor axes of the apertures which we use are twice the FWHM values of the source listed in Table 1, and hence the smallest of our sources have their fluxes measured in apertures with minor axes $\sim 40$ arcsec. Hence, for the smallest of our sources, the $850-\mu \mathrm{m}$ flux density may be underestimated by up to a maximum of 10 per cent. However, for the large majority of our sources, the aperture photometry correction should be $\lesssim 3$ per cent. We direct the reader to Dempsey et al. (2013) for further information.

The SED of each source was fitted with a modified blackbody distribution (see Fig. 5), in order to determine the mean, columndensity-weighted, line-of-sight dust temperature. The monochromatic flux density $F_{v}$ is given at frequency $v$ by

$\lambda F_{\lambda}=v F_{v}=v \Omega f B_{v}(T)\left(1-\mathrm{e}^{-\left(\frac{v}{v_{\mathrm{c}}}\right)^{\beta}}\right)$,

where $B_{v}(T)$ is the Planck function at dust temperature $T, \Omega$ is the solid angle of the aperture, $f$ is the filling factor of the source in the aperture, and $\beta$ is the dust emissivity index. The frequency at which the optical depth becomes unity is taken to be the canonical value, $v_{\mathrm{c}}=6 \mathrm{THz}$ (Ward-Thompson, André \& Kirk 2002), as we do not have enough data points to accurately constrain a fourth parameter in our fitting process.

We determined a typical dust emissivity index $(\beta)$, which we adopted for our cores in order to more accurately constrain the SED fitting process. While temperature and dust emissivity index can be fitted simultaneously using SCUBA-2 $850 \mu \mathrm{m}$ data in conjunction 
Table 1. Sources found by the CSAR algorithm in its non-hierarchical mode in the $850-\mu m$ SCUBA-2 data - see text for details. Sources labelled 'S', listed in this table, are detected in SCUBA-2 $850 \mu \mathrm{m}$ emission. See text for details.

\begin{tabular}{|c|c|c|c|c|c|c|c|c|c|c|}
\hline $\begin{array}{l}\text { Source } \\
\text { Index }\end{array}$ & $\begin{array}{c}\text { RA } \\
(\mathrm{J} 2000)\end{array}$ & $\begin{array}{c}\text { Dec. } \\
(\mathrm{J} 2000)\end{array}$ & $\begin{array}{c}\text { FWHM } \\
(\operatorname{arcsec} \times \operatorname{arcsec})\end{array}$ & $\begin{array}{c}\text { Angle } \\
\left({ }^{\circ} \mathrm{E} \text { of } \mathrm{N}\right)\end{array}$ & $160 \mu \mathrm{m}$ & $250 \mu \mathrm{m}$ & $\begin{array}{l}F_{v}^{\text {total }}(\mathrm{Jy}) \\
350 \mu \mathrm{m}\end{array}$ & $500 \mu \mathrm{m}$ & $850 \mu \mathrm{m}$ & $\begin{array}{c}\text { Counterpart } \\
\text { sources }\end{array}$ \\
\hline S1 & 4:18:40.00 & $+28: 23: 15.6$ & $57.0 \times 39.0$ & 84.0 & 30.99 & 45.27 & 32.86 & 18.70 & 5.21 & $\mathrm{H} 1, \mathrm{~F} 1$ \\
\hline $\mathrm{S} 2$ & $4: 17: 42.10$ & $+28: 08: 44.4$ & $54.6 \times 21.4$ & 167.0 & 0.74 & 3.15 & 3.80 & 2.79 & 1.08 & H26, F15 \\
\hline S3 & $4: 17: 50.18$ & $+27: 56: 05.5$ & $53.4 \times 44.2$ & 93.0 & 2.39 & 7.51 & 7.80 & 5.44 & 2.07 & $\mathrm{H} 47, \mathrm{~F} 14$ \\
\hline $\mathrm{S} 4$ & $4: 13: 48.01$ & $+28: 12: 38.3$ & $26.4 \times 24.1$ & 74.0 & 1.11 & 3.00 & 3.13 & 2.47 & 0.80 & H6, F9 \\
\hline S5 & 4:18:08.17 & $+28: 05: 10.3$ & $39.6 \times 32.0$ & 121.0 & 0.96 & 3.69 & 4.30 & 3.43 & 1.40 & $\mathrm{H} 40, \mathrm{~F} 16$ \\
\hline S6 & $4: 18: 03.83$ & $+28: 23: 03.5$ & $30.0 \times 23.3$ & 33.0 & 0.93 & 2.17 & 2.19 & 1.60 & 0.62 & H7, F12 \\
\hline S7 & $4: 17: 43.31$ & $+28: 06: 04.5$ & $32.4 \times 20.7$ & 45.0 & 0.48 & 1.60 & 1.70 & 1.22 & 0.51 & H28, F17 \\
\hline S8 & $4: 17: 01.36$ & $+28: 26: 36.0$ & $54.6 \times 46.0$ & 66.0 & 5.28 & 8.93 & 6.69 & 3.99 & 2.29 & $\mathrm{H} 15, \mathrm{~F} 8$ \\
\hline S9 & $4: 18: 11.60$ & $+27: 35: 54.4$ & $54.0 \times 36.9$ & 11.0 & 2.19 & 5.80 & 6.22 & 4.58 & 1.65 & H41, F19 \\
\hline S10 & $4: 14: 27.63$ & $+28: 07: 11.6$ & $55.2 \times 26.2$ & 106.0 & 5.19 & 8.56 & 6.45 & 3.82 & 1.03 & H8, F4 \\
\hline $\mathrm{S} 11$ & 4:18:03.08 & $+28: 07: 35.2$ & $39.0 \times 20.5$ & 126.0 & 0.30 & 1.28 & 1.57 & 1.24 & 0.69 & None \\
\hline $\mathrm{S} 12$ & $4: 17: 34.58$ & $+28: 03: 05.0$ & $55.2 \times 20.3$ & 53.0 & 1.98 & 4.39 & 3.85 & 2.51 & 0.74 & H19, F10 \\
\hline S13 & $4: 14: 27.53$ & $+28: 04: 58.1$ & $45.6 \times 20.8$ & 158.0 & 3.20 & 4.76 & 3.28 & 1.89 & 0.64 & $\mathrm{H} 11, \mathrm{~F} 5$ \\
\hline S14 & $4: 17: 52.08$ & $+28: 12: 31.1$ & $51.6 \times 48.7$ & 93.0 & 2.21 & 5.24 & 5.28 & 4.08 & 1.75 & $\mathrm{H} 23, \mathrm{~F} 18$ \\
\hline $\mathrm{S} 15$ & $4: 14: 12.33$ & $+28: 08: 46.6$ & $36.0 \times 18.4$ & 7.0 & 2.24 & 2.78 & 2.06 & 1.17 & 0.27 & H9, F6 \\
\hline S16 & $4: 18: 33.13$ & $+28: 27: 31.0$ & $85.8 \times 24.0$ & 172.0 & 1.03 & 3.17 & 3.34 & 2.34 & 0.91 & None \\
\hline S17 & $4: 16: 57.34$ & $+28: 29: 35.6$ & $54.6 \times 32.0$ & 38.0 & 1.44 & 2.96 & 2.61 & 1.84 & 1.04 & H58, F22 \\
\hline S18 & $4: 17: 42.09$ & $+27: 39: 15.0$ & $70.8 \times 26.0$ & 75.0 & 2.70 & 4.94 & 4.01 & 2.97 & 0.86 & H13, F13 \\
\hline S19 & $4: 18: 00.55$ & $+28: 11: 08.7$ & $45.0 \times 22.2$ & 165.0 & 0.55 & 1.70 & 1.74 & 1.23 & 0.52 & H45, F43 \\
\hline S20 & $4: 17: 52.87$ & $+28: 23: 40.0$ & $44.4 \times 37.1$ & 160.0 & 1.16 & 2.08 & 1.78 & 1.28 & 0.89 & H18, F36 \\
\hline S21 & $4: 17: 37.42$ & $+28: 12: 06.0$ & $51.0 \times 35.4$ & 27.0 & 1.40 & 3.06 & 2.61 & 1.67 & 0.73 & $\mathrm{H} 44, \mathrm{~F} 21$ \\
\hline $\mathrm{S} 22$ & $4: 17: 50.02$ & $+27: 37: 56.1$ & $38.4 \times 28.8$ & 140.0 & 0.58 & 1.31 & 1.09 & 0.81 & 0.36 & H34, F46 \\
\hline $\mathrm{S} 23$ & $4: 18: 52.50$ & $+28: 20: 23.0$ & $44.4 \times 24.8$ & 99.0 & 5.47 & 4.95 & 2.75 & 1.53 & 0.33 & H3, F3 \\
\hline S24 & 4:18:07.41 & $+28: 24: 48.7$ & $70.8 \times 30.9$ & 30.0 & 1.92 & 3.87 & 3.15 & 1.94 & 0.64 & $\mathrm{H} 10, \mathrm{~F} 20$ \\
\hline S25 & $4: 17: 20.14$ & $+28: 18: 56.5$ & $52.8 \times 40.1$ & 12.0 & 2.76 & 3.84 & 2.51 & 1.42 & 0.71 & H16, F27 \\
\hline
\end{tabular}

with the Herschel photometric bands (see Sadavoy et al. 2013), the Herschel data alone, covering the wavelength range 160-500 $\mu \mathrm{m}$, do not provide the long-wavelength information necessary to accurately constrain both parameters. We decided to use a fixed value of $\beta$ when deriving best-fitting temperatures for all of our cores, including those with an $850-\mu \mathrm{m}$ detection, in order to make a fair comparison between the different sets of cores. In order to find a suitable $\beta$, we fitted SEDs to the subset of the filtered-Herschel cores with detections in 850- $\mu \mathrm{m}$ emission. We determined an SED using only the filtered Herschel fluxes for each source, and from the best-fitting SED predicted an $850-\mu \mathrm{m}$ flux density. These predicted $850-\mu \mathrm{m}$ flux densities were then compared to the values measured in the SCUBA-2 850- $\mu \mathrm{m}$ map. We repeated this process for a range of values of $\beta$, as well as allowing $\beta$ to vary as a free parameter, in order to determine the value that best predicted the $850-\mu \mathrm{m}$ flux densities.

For each of the values of $\beta$ tested, we calculated the mean reduced $\chi^{2}$ value of the SED fits. We also calculated the reduced $\chi^{2}$ value of the 1:1 relation between predicted and measured $850-\mu \mathrm{m}$ flux densities. We found that when emissivity index was allowed to vary as a free parameter, the $850-\mu \mathrm{m}$ flux densities were generally wellpredicted. We found the mean value of $\beta$ when it was varied as a free parameter to be 1.3 , and the standard deviation on this value to be 0.6 . When $\beta$ was fixed, we found that values in the range $\beta$ $=1.1-1.4$ gave indistinguishably good results. We therefore chose to adopt a value of dust emissivity index $\beta=1.3 \pm 0.6$ for the remainder of this work.

A dust emissivity index of 1.3 is lower than that typically expected for a starless core. A wide variety of $\beta$ values have been determined for starless cores. For example, Shirley et al. (2005) found $\beta=1.8-1.9$ for the starless core L1498; Friesen et al. (2005) found $\beta=1.3-2.1$ for a sample of hot starless cores; Schnee et al. (2010) found $\beta=2.2 \pm 0.6$ for the starless core TMC- $1 \mathrm{c}$; and $\mathrm{Sa}-$ davoy et al. (2013) found $\beta=1.6-2.0$ towards cores in the Perseus molecular cloud. Our low value of $\beta$ may be due to there being multiple dust temperature components along the lines of sight towards our sources, which would broaden the SEDs of the sources, and hence lower their apparent $\beta$ values. This effect was discussed in detail by Martin et al. (2012). Starless cores are not expected to be isothermal sources: a temperature gradient from $15 \mathrm{~K}$ at their edges to $7 \mathrm{~K}$ in their heavily shielded centres has been seen elsewhere (e.g. Stamatellos, Whitworth \& Ward-Thompson 2007), even in the absence of external heating sources. The surrounding, more tenuous, material of the molecular cloud will be warmer still. While the spatial filtering of the SCUBA-2 observations will to some extent ameliorate this effect, there will still be some material along the line of sight not associated with the core.

Our low $\beta$ might alternatively, or additionally, be the result of grain growth within the densest regions of the starless cores. Our value of $\beta$ is intermediate between the value expected in molecular clouds of $\beta=1.5-2.0$ (e.g. Draine \& Lee 1984; Draine et al. 2007), and $\beta=1.0$, expected in protoplanetary discs (Beckwith \& Sargent 1991).

We fitted our sources using $\beta=1.3$ in order to determine their dust temperatures. We then determined our source masses using the Hildebrand (1983) relation

$M=\frac{F_{v}(850 \mu \mathrm{m}) D^{2}}{\kappa_{v(850 \mu \mathrm{m})} B_{v(850 \mu \mathrm{m})}(T)}$,

where $F_{v}(850 \mu \mathrm{m})$ is the flux density at $850 \mu \mathrm{m}$. For the SCUBA2 sources, $F_{v}(850 \mu \mathrm{m})$ was taken to be the measured SCUBA-2 $850-\mu \mathrm{m}$ flux density of the source, while for the Herschel and 

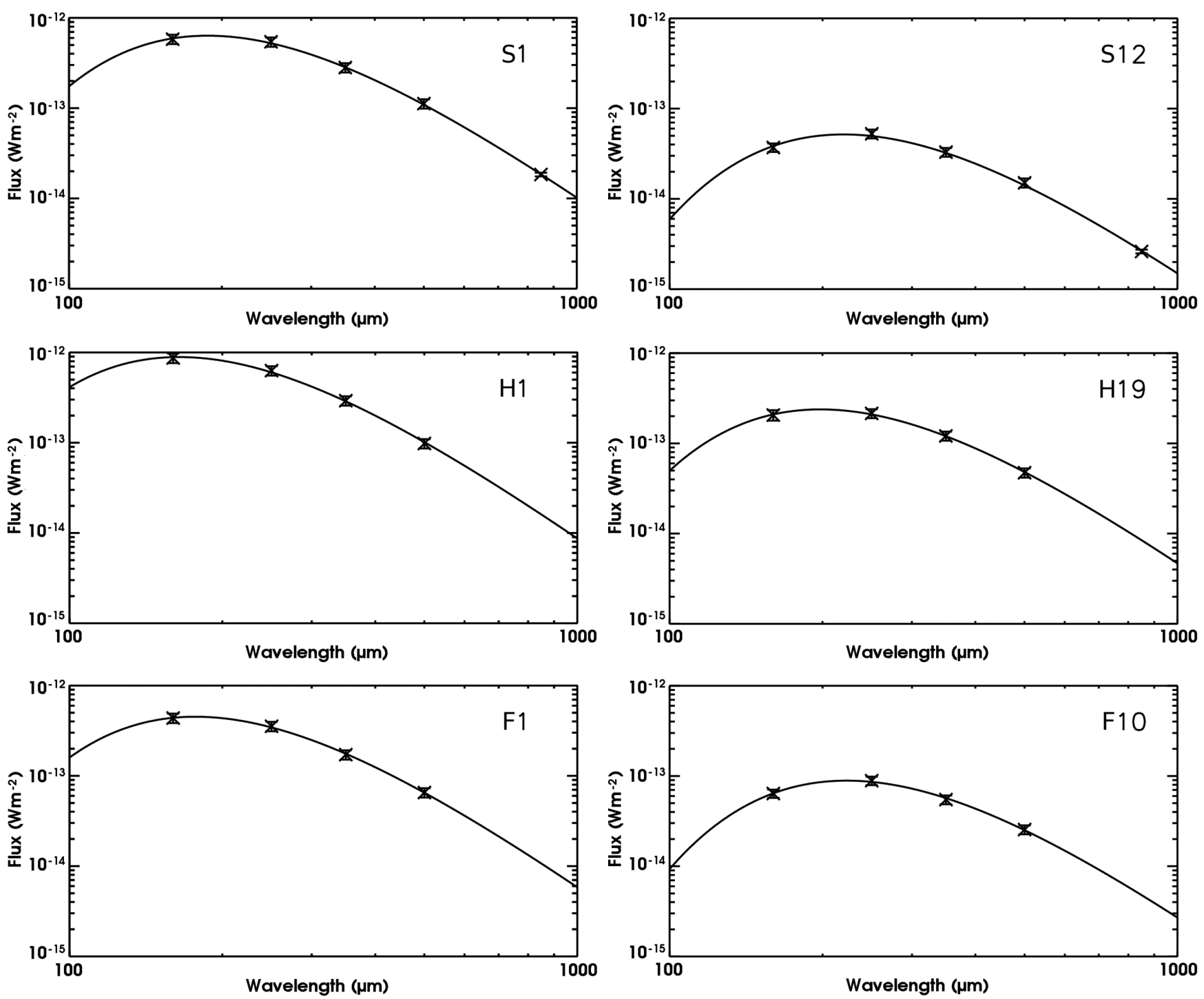

Figure 5. SED fits for sources S1 and S12, and their counterparts in the Herschel and filtered-Herschel catalogues (H1 and F1, and H19 and F10, respectively). See text for details.

filtered-Herschel sources, $F_{v}(850 \mu \mathrm{m})$ was taken to be the flux density at $850 \mu \mathrm{m}$ as extrapolated from the best-fitting SED. $D$ is the distance to Taurus $(140 \mathrm{pc}), B_{v(850 \mu \mathrm{m})}(T)$ is the Planck function, and $\kappa_{v(850 \mu \mathrm{m})}$ is the dust mass opacity, as parametrized by Beckwith et al. (1990): $\kappa_{v}=0.1\left(v / 10^{12} \mathrm{~Hz}\right)^{\beta} \mathrm{cm}^{2} \mathrm{~g}^{-1}$ (assuming a standard dust-to-gas mass ratio of 1:100). Again, the dust emissivity index $\beta$ was taken to be 1.3. Some example SED fits for sources with counterparts in all three catalogues are shown in Fig. 5.

We calculated the mean volume density of molecular hydrogen for each source as

$n\left(\mathrm{H}_{2}\right)=\frac{M}{\mu m_{\mathrm{H}}} \frac{1}{\frac{4}{3} \pi R^{3}}$,

where $R$ is the equivalent deconvolved FWHM of the source. The equivalent FWHM was taken to be the geometric mean of the deconvolved major and minor FWHMs, as determined by CSAR. The deconvolution assumed a beam size of 14.1 arcsec in the case of SCUBA-2 sources and 18.1 arcsec in the case of Herschel and filtered-Herschel sources. The mean molecular weight $\mu$ was taken to be 2.86 , assuming that the gas is $\sim 70$ per cent $\mathrm{H}_{2}$ by mass (Kirk et al. 2013).

\section{DISCUSSION}

The temperatures, masses, densities and sizes of the cores detected by SCUBA-2 are listed in Table 2. The same information for Herschel and filtered-Herschel sources is listed in Tables A3 and A4 in the online appendix. The properties of those sources with counterparts in another catalogue are compared with the properties of their counterparts in Figs 6 and 7. A source's counterpart in another catalogue is the nearest neighbour to the source in that catalogue, provided that the source and its neighbour are separated by less than the FWHM of the larger of the two sources. It can be seen from the central column of plots in Figs 6 and 7 that sources found in both the SCUBA-2 and the filtered-Herschel catalogues are typically found to have very similar properties. This tends to indicate that the filtering process is the correct method by which to make SCUBA-2 and Herschel data comparable. 
Table 2. Derived properties of the sources found by SCUBA-2 in the L1495 region - temperature, mass, density and mean deconvolved full-width at half-maximum (geometric mean of major and minor axes with beam-size subtracted in quadrature).

\begin{tabular}{lcccc}
\hline $\begin{array}{c}\text { Source } \\
\text { index }\end{array}$ & $\begin{array}{c}\text { Temp. } \\
(\mathrm{K})\end{array}$ & $\begin{array}{c}\text { Mass } \\
\left(\mathrm{M}_{\odot}\right)\end{array}$ & $\begin{array}{c}\mathrm{H}_{2} \\
\text { density } \\
\left(\times 10^{4} \mathrm{~cm}^{-3}\right)\end{array}$ & $\begin{array}{c}\text { Deconv. } \\
\text { FWH } \\
(\mathrm{pc})\end{array}$ \\
\hline S1 & $14.9 \pm 0.4$ & $0.613 \pm 0.267$ & $6.34 \pm 2.76$ & 0.032 \\
S2 & $10.1 \pm 0.2$ & $0.256 \pm 0.057$ & $6.94 \pm 1.56$ & 0.023 \\
S3 & $10.9 \pm 0.2$ & $0.420 \pm 0.114$ & $3.96 \pm 1.08$ & 0.033 \\
S4 & $11.2 \pm 0.2$ & $0.156 \pm 0.034$ & $10.55 \pm 2.29$ & 0.017 \\
S5 & $10.1 \pm 0.2$ & $0.336 \pm 0.081$ & $8.08 \pm 1.94$ & 0.024 \\
S6 & $11.2 \pm 0.2$ & $0.120 \pm 0.025$ & $7.04 \pm 1.48$ & 0.018 \\
S7 & $10.6 \pm 0.2$ & $0.110 \pm 0.023$ & $6.88 \pm 1.41$ & 0.018 \\
S8 & $12.0 \pm 0.3$ & $0.391 \pm 0.116$ & $3.37 \pm 1.00$ & 0.034 \\
S9 & $11.1 \pm 0.2$ & $0.324 \pm 0.082$ & $3.95 \pm 1.00$ & 0.030 \\
S10 & $14.4 \pm 0.4$ & $0.126 \pm 0.029$ & $2.47 \pm 0.58$ & 0.026 \\
S11 & $9.3 \pm 0.2$ & $0.196 \pm 0.043$ & $9.38 \pm 2.04$ & 0.019 \\
S12 & $12.7 \pm 0.3$ & $0.113 \pm 0.024$ & $3.27 \pm 0.70$ & 0.023 \\
S13 & $14.3 \pm 0.4$ & $0.079 \pm 0.017$ & $2.94 \pm 0.64$ & 0.021 \\
S14 & $10.9 \pm 0.2$ & $0.355 \pm 0.092$ & $3.05 \pm 0.79$ & 0.034 \\
S15 & $16.1 \pm 0.5$ & $0.026 \pm 0.005$ & $1.67 \pm 0.34$ & 0.017 \\
S16 & $10.9 \pm 0.2$ & $0.182 \pm 0.039$ & $2.11 \pm 0.45$ & 0.031 \\
S17 & $11.0 \pm 0.2$ & $0.205 \pm 0.047$ & $3.03 \pm 0.70$ & 0.028 \\
S18 & $12.9 \pm 0.3$ & $0.124 \pm 0.027$ & $1.69 \pm 0.37$ & 0.029 \\
S19 & $10.7 \pm 0.2$ & $0.109 \pm 0.022$ & $3.72 \pm 0.76$ & 0.021 \\
S20 & $10.7 \pm 0.2$ & $0.186 \pm 0.042$ & $3.02 \pm 0.69$ & 0.028 \\
S21 & $11.9 \pm 0.2$ & $0.122 \pm 0.026$ & $1.73 \pm 0.37$ & 0.029 \\
S22 & $11.4 \pm 0.2$ & $0.065 \pm 0.013$ & $1.90 \pm 0.38$ & 0.023 \\
S23 & $19.6 \pm 0.7$ & $0.023 \pm 0.005$ & $0.69 \pm 0.15$ & 0.023 \\
S24 & $12.9 \pm 0.3$ & $0.090 \pm 0.018$ & $0.95 \pm 0.20$ & 0.032 \\
S25 & $13.4 \pm 0.3$ & $0.095 \pm 0.021$ & $1.05 \pm 0.23$ & 0.031 \\
\hline & & & &
\end{tabular}

Herschel sources are typically measured to be warmer than their SCUBA-2 and filtered-Herschel counterparts. We hypothesize that this is due to the filtering process removing much of the extended foreground and background emission originating from warmer material, thus reducing the line-of-sight temperature determined by the fitting process. Source FWHM size is typically not strongly affected by the filtering process, with no clear propensity for cores to become smaller or larger. This suggests that CSAR is identifying the core material accurately: in the unfiltered case CSAR is identifying emission peaks above the background large-scale emission of the cloud, while in the filtered case, the background large-scale emission has been removed, and CSAR is identifying emission peaks above the noise in the data.

Herschel sources are typically more massive than their counterpart filtered-Herschel sources. Again, this is due to the removal of large-scale signal by the filtering process. Without the additional contribution of the background signal, the total flux measured in the filtered-Herschel apertures will be less than that measured in corresponding Herschel apertures (typically similarly sized, as discussed above).

Herschel sources are typically denser than their counterpart filtered-Herschel sources. This is a counterintuitive result, as the removal of low-density large-scale structure might be expected to leave behind only the higher-density cores. However, as discussed above, the size of the cores detected by CSAR is largely unaffected by the filtering process, which is likely due to CSAR detecting the core itself, rather than the material in which it is embedded. Hence, a given core detected in both Herschel and filtered-Herschel emission will be of the same size in both cases, but will be less massive, and so less dense, in the filtered-Herschel case.
These results suggest that the measures of core temperature, mass and density determined from the filtered-Herschel data are more representative of the true properties of the starless cores than those determined from the unfiltered Herschel data, as in the filteredHerschel case the flux measured should be that of the core itself, rather than being the flux of both the core and the full line-of-sight column of material in which it is embedded.

Fig. 8 shows the relationship between temperature and density for the filtered-Herschel sources and the SCUBA-2 sources. The SCUBA-2 sources follow the same density-temperature relation as the filtered-Herschel sources, $n\left(\mathrm{H}_{2}\right) \propto T^{-8.5 \pm 0.5}$. However, only the densest filtered-Herschel sources have a counterpart SCUBA-2 source, with the minimum SCUBA-2 source density being $\sim 3 \times 10^{-17} \mathrm{~kg} \mathrm{~m}^{-3}\left(\sim 6 \times 10^{3} \mathrm{H}_{2} / \mathrm{cm}^{3}\right)$, while the lowest-density filtered-Herschel sources are $\sim 2 \times 10^{-18} \mathrm{~kg} \mathrm{~m}^{-3}$ $\left(\sim 4 \times 10^{2} \mathrm{H}_{2} / \mathrm{cm}^{3}\right)$. The filtered-Herschel sources and the SCUBA-2 sources have a similar range of temperatures, being $~ 9-20 \mathrm{~K}$.

Those SCUBA-2 sources which do not follow the temperaturedensity relation are S1 (L1495A-S), S10, S13, S15 and S23. All of these sources are significantly $(\sim 5 \mathrm{~K})$ warmer than might be expected from their density. Of these five sources, S1 and S23 are heated by V892 Tau (IRAS 04155+2812), as discussed above. Sources S10 and S13 are being heated by IRAS $04113+2758$, while source S15 is associated with the IR source IRAS $04111+2800 \mathrm{G}$, and may in fact have an embedded YSO within it, possibly a VeLLO (Very Low Luminosity Object - Young et al. 2004).

Fig. 9 shows the mass-size relation for the filtered-Herschel and SCUBA-2 sources. There is no tendency for the SCUBA-2 sources to be smaller in size than the filtered-Herschel sources. However, the SCUBA-2 sources are among the most massive. The grey band shown in Fig. 9 indicates the region of the mass/size plane which unbound, transient starless cores are expected to inhabit (Elmegreen \& Falgarone 1996; André et al. 2010). A substantial fraction of the filtered-Herschel sources lie within this region. However, the SCUBA-2 sources in almost all cases occupy the region of the mass/size plot in which gravitationally bound prestellar cores are expected to be found (cf. André et al. 2010).

Fig. 8 shows a clear cut-off in density below which no SCUBA-2 sources are detected. However, there appears to be no similar cut-off in temperature. Fig. 9 shows no tendency for SCUBA-2 sources to be smaller in radius than filtered-Herschel sources. This indicates that the criterion for determining whether a source detected using Herschel will be detectable with SCUBA-2 is its mass for a given size or, in other words, density.

This corresponds, in terms of the measurable parameters, effectively to surface brightness. For a given temperature, the higher column density material will produce a higher surface brightness. Also, for non-elongated geometries, higher column density corresponds to higher volume density. Therefore, it appears that it is not mass or size alone that determines detectability with SCUBA-2, but rather a combination of mass and size that corresponds to density. Dust at temperatures typically found in starless cores emits more flux at Herschel wavelengths than at SCUBA2 850- $\mu \mathrm{m}$ wavelength, because Herschel wavelengths coincide with the peak of the blackbody function, while $850 \mu \mathrm{m}$ is on the Rayleigh-Jeans tail. Hence, for roughly the same absolute noise level (which these maps have), any given core will appear at a higher signal-to-noise ratio to Herschel than to SCUBA-2. The exceptions to this are those sources with associated or nearby stars or protostars, because in those cases the above assumption, that everything is at roughly similar temperature, breaks down. 


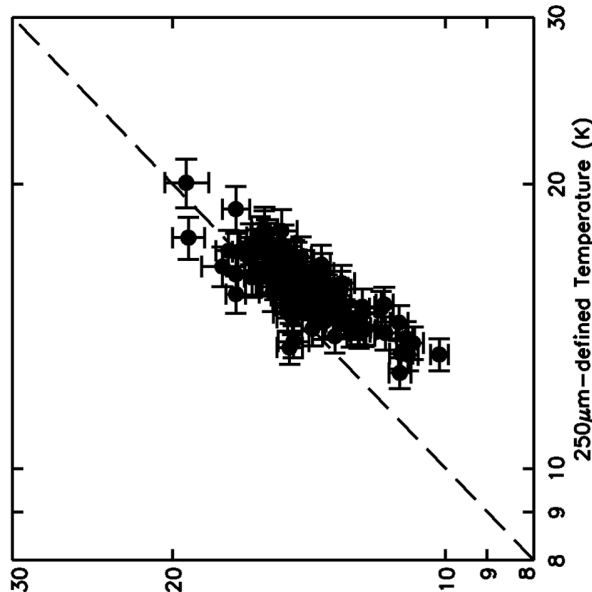

(ㄱ)

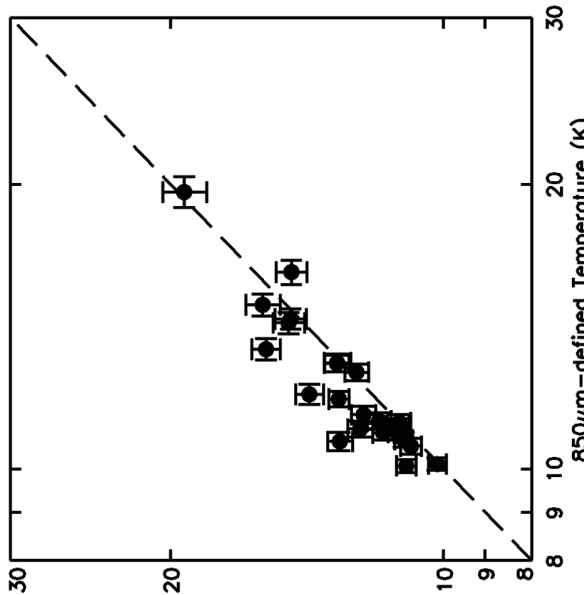

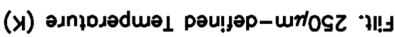

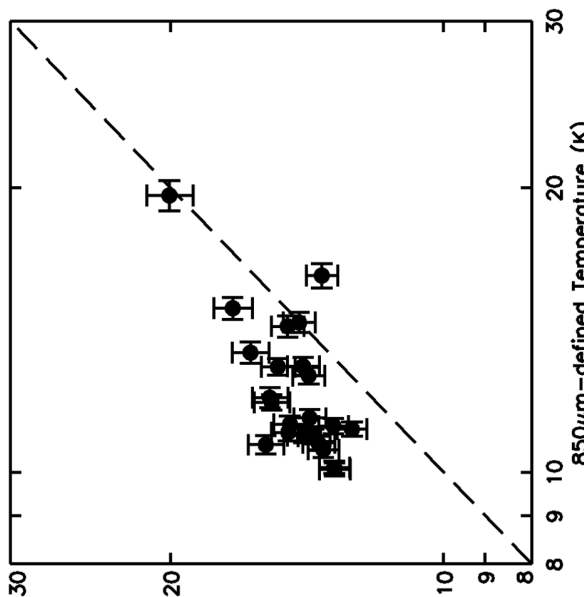

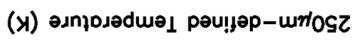
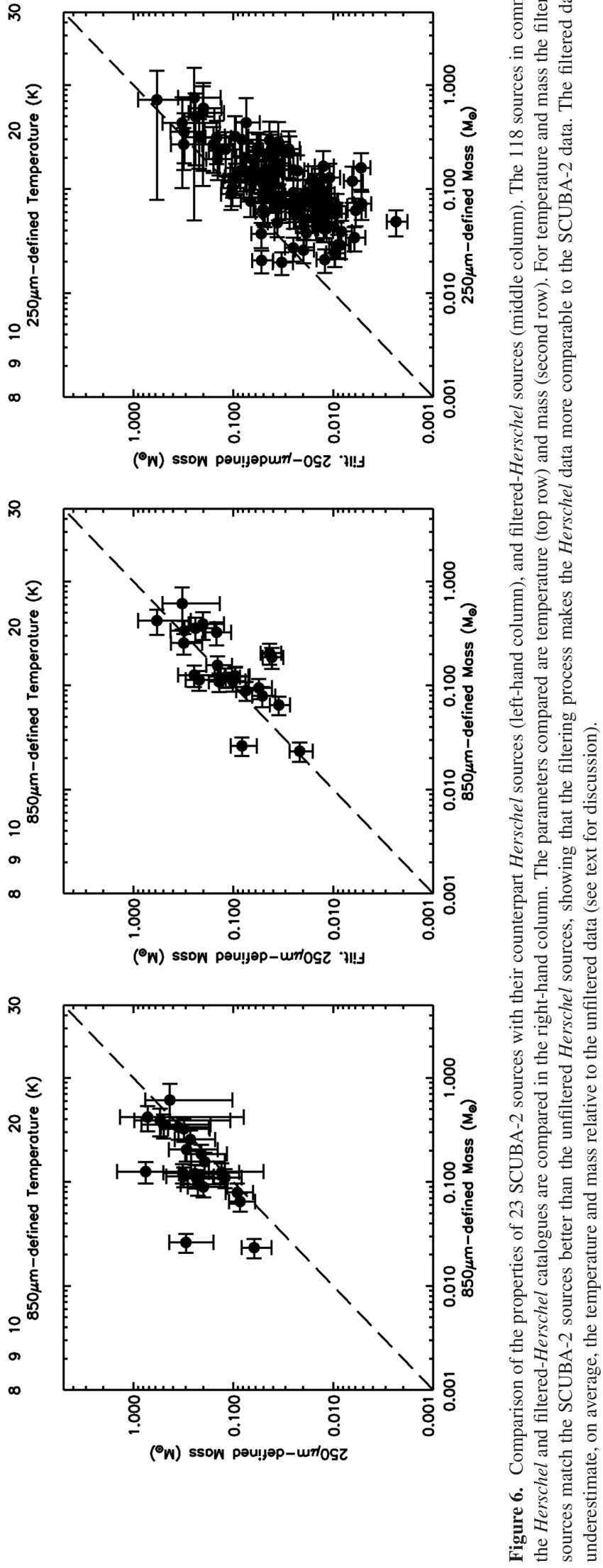

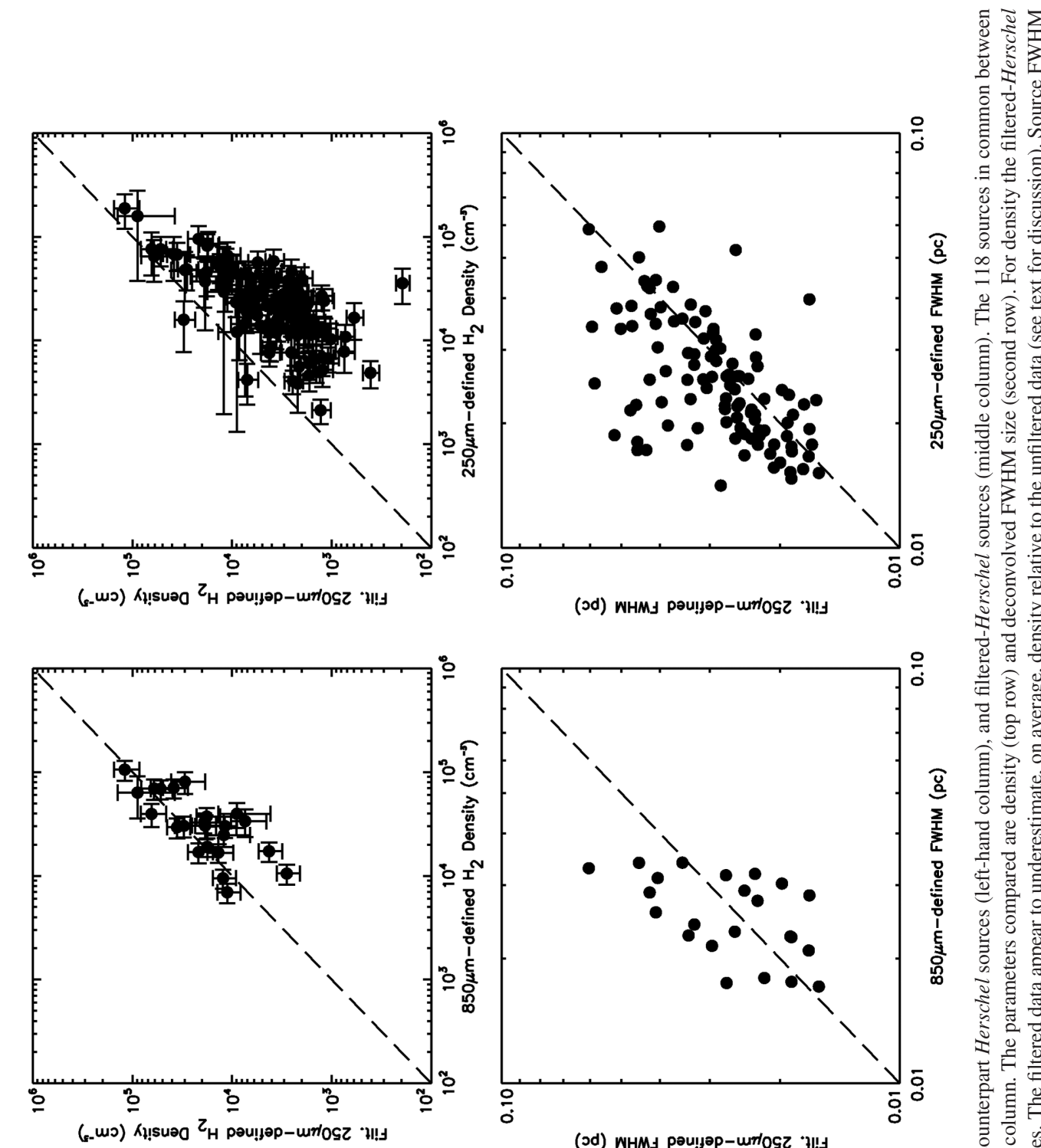

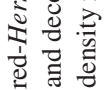

(o) WHMJ pau!gap-urrosz
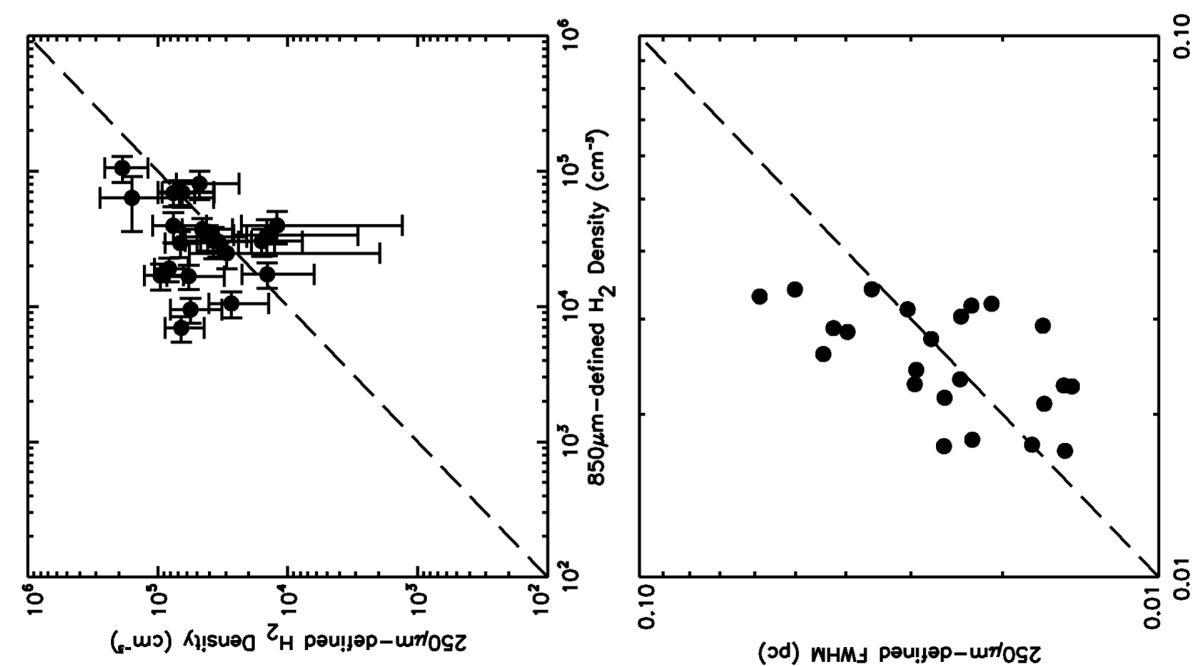

(od) WHMJ pəu!yep-wriosz 


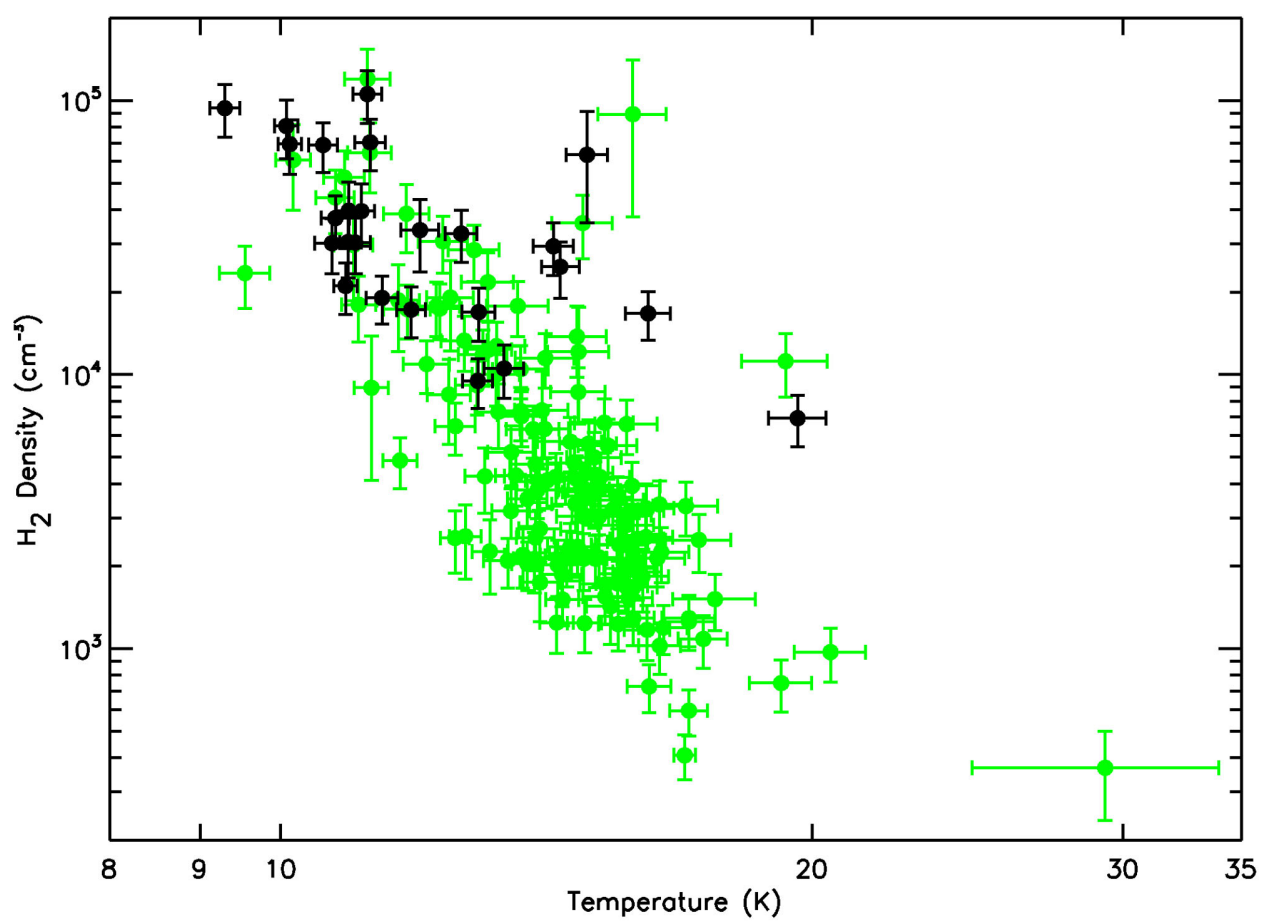

Figure 8. Plot of core density against temperature. Black symbols are SCUBA-2 sources, green symbols are filtered-Herschel sources. There appears to be a cut-off in density, with the minimum SCUBA-2 source density being $\sim 6 \times 10^{3} \mathrm{H}_{2} / \mathrm{cm}^{3}$, while the lowest-density filtered-Herschel sources are $\sim 4 \times 10^{2}$ $\mathrm{H}_{2} / \mathrm{cm}^{3}$. However, the filtered-Herschel sources and the SCUBA-2 sources have a similar range in temperature, $\sim 9-20 \mathrm{~K}$, with no apparent cut-off.

We test this hypothesis further in Appendix B by scaling the rms noise on the filtered-Herschel data such that the relative, rather than the absolute, noise levels of the SCUBA-2 850- $\mu \mathrm{m}$ and filteredHerschel $250-\mu \mathrm{m}$ data are comparable. We show in Appendix B that the sources detected in the increased-noise filtered-Herschel data are preferentially the denser sources, as expected.

Fig. 10 shows how the minimum density $n$ to which SCUBA-2 is sensitive varies as a function of distance and temperature. A core of temperature $T$ at distance $D$ will be detectable in SCUBA-2 GBS data if its density is greater than or equal to $n$, where

$n=n_{0}\left(\frac{D}{D_{0}}\right)^{2} \frac{\mathrm{e}^{\frac{h v}{k^{T}}}-1}{\mathrm{e}^{\frac{h v}{k^{B^{T}}}}-1}$.

This relation is normalized to a density sensitivity $n_{0}=6.3 \times 10^{3}$ $\mathrm{H}_{2} / \mathrm{cm}^{3}$ at the canonical distance of the Taurus molecular cloud of $D_{0}=140 \mathrm{pc}$ and the mean temperature of our non-externally heated starless cores, $T_{0}=11.3 \mathrm{~K}$. The density sensitivity limit at a given distance decreases as source temperature increases.

The distances to the Taurus and Orion molecular clouds are marked in Fig. 10. The density sensitivity limits for a $10 \mathrm{~K}$ core at $140 \mathrm{pc}$ (close to the typical core temperature in Taurus) and a $50 \mathrm{~K}$ core at $450 \mathrm{pc}$ (a typical core temperature in the high-mass star-forming region Orion, shown for comparison) are very similar: $8 \times 10^{3} \mathrm{H}_{2} / \mathrm{cm}^{3}$ and $7 \times 10^{3} \mathrm{H}_{2} / \mathrm{cm}^{3}$, respectively. Hence, their relative detectability in SCUBA-2 GBS data should be similar.

It is important to note that the SCUBA-2 sensitivity limits found in this work are those of the JCMT GBS Internal Release 1 data reduction, and not an intrinsic property of data from the instrument. It is possible for further data to be taken, improving the sensitivity, until the confusion limit is reached. Similarly, the Herschel data are at the (fixed) sensitivity of the HGBS, and not necessarily confusionlimited. Furthermore, as discussed above, we employed a stringent set of source selection criteria when creating our catalogue. However, this does not alter our conclusion that, for SCUBA-2 and Herschel maps treated identically and with sources extracted in the same manner, the surface brightness effect from which the density sensitivity threshold results will result in SCUBA-2 picking out the densest subset of the cores detected by Herschel, and hence those most likely to be pre-stellar in nature.

\section{CONCLUSIONS}

In this paper we have compared data of the Taurus L1495 region from JCMT-SCUBA-2 at $850 \mu \mathrm{m}$ with Herschel data from 160 to $500 \mu \mathrm{m}$. We have spatially filtered the Herschel data to match the data processing carried out on the SCUBA- 2 data.

We have extracted, and characterized, the properties of, starless and pre-stellar cores from the SCUBA-2 $850-\mu \mathrm{m}$, Herschel-SPIRE 250- $\mu \mathrm{m}$, and spatially filtered Herschel $250-\mu \mathrm{m}$ data, and have compared the cores found in the different data sets. Our goal was to determine which property of a starless core identified by Herschel is most important in determining whether the same core would be detected with SCUBA-2.

We identified sources using the CSAR extraction algorithm. We detected 25 sources in the SCUBA-2 850- $\mu$ m data, 208 sources in the Herschel $250-\mu \mathrm{m}$ data, and 138 sources in the spatially filtered Herschel $250-\mu \mathrm{m}$ data.

We determined a representative dust emissivity index of our sources of $\beta=1.3 \pm 0.6$. This was the value of $\beta$ which best predicted the SCUBA-2 850- $\mu$ m flux densities of our sources from their SED in filtered Herschel emission. We determined mean lineof-sight temperatures for our sources by SED fitting. This then allowed an accurate mass determination to be made for each source.

We found that cores detected by SCUBA- 2 and cores detected in filtered $250-\mu \mathrm{m}$ emission have similar properties, obeying the same 


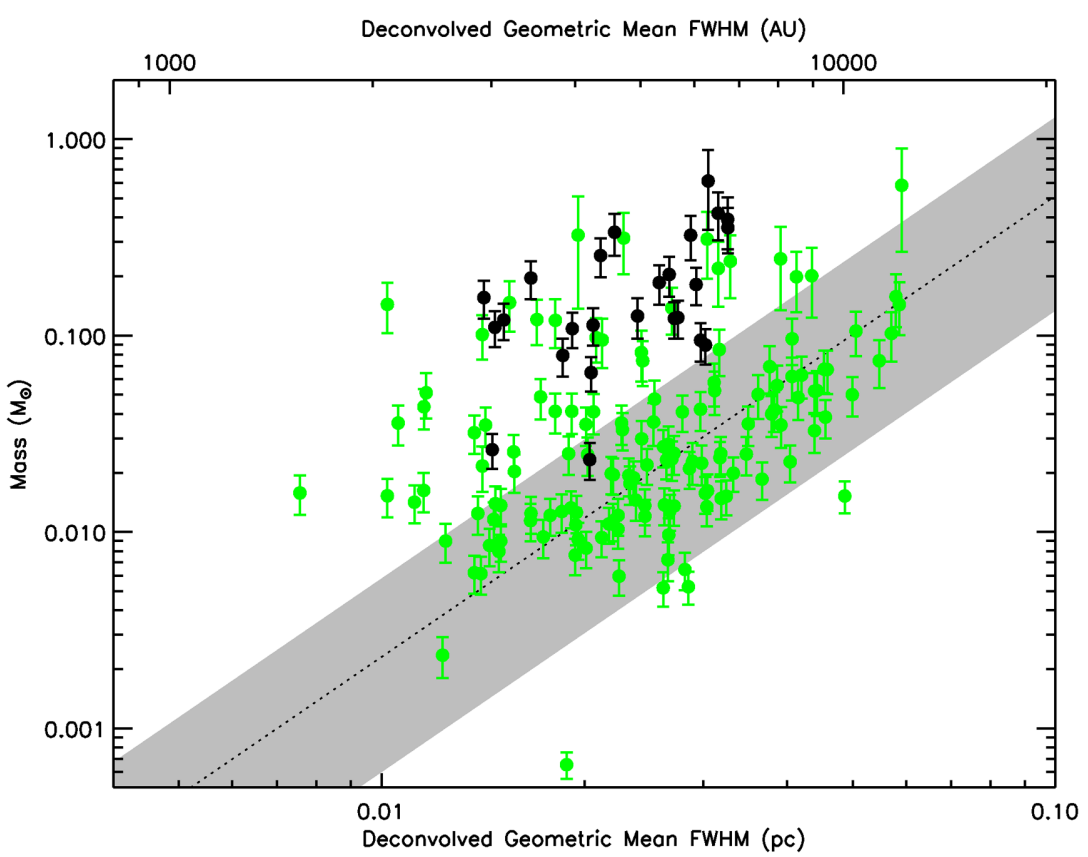

Figure 9. Plot of core mass against deconvolved size. Black symbols are SCUBA-2 sources, green symbols are filtered-Herschel sources. There appears to be no tendency for the SCUBA-2 sources to be smaller in size than the filtered-Herschel sources. However, the SCUBA-2 sources are among the most massive. The grey band indicates the region which unbound starless cores are expected to inhabit (Elmegreen \& Falgarone 1996; André et al. 2010). A substantial fraction of the filtered-Herschel sources lie within this region. However, the SCUBA-2 sources in almost all cases occupy the region above this, in which prestellar cores are expected to be found (cf. André et al. 2010).

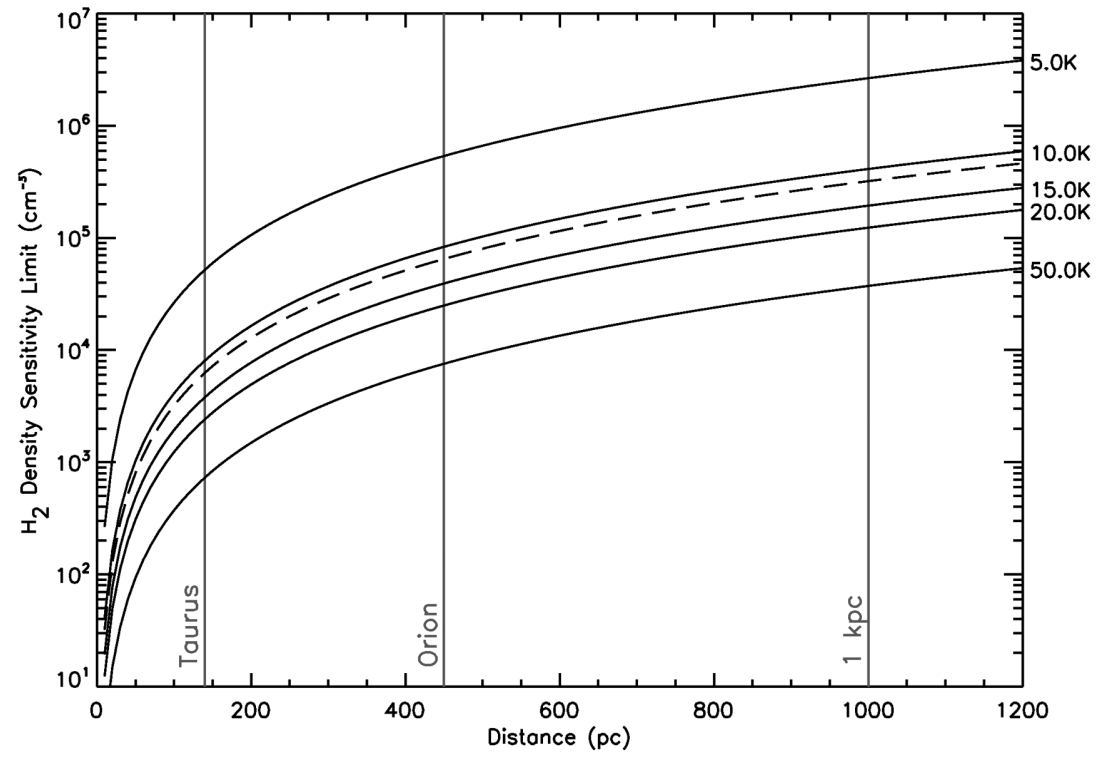

Figure 10. Plot of the lowest source density detectable using SCUBA-2 Internal Release 1 GBS data, as a function of distance, for various assumed source temperatures. The dashed line shows the SCUBA-2 GBS density sensitivity limit as a function of distance at the mean temperature of the non-externally heated starless cores in our sample $(11.3 \mathrm{~K})$. All of the relations are normalized against a density limit of $\sim 6.3 \times 10^{3} \mathrm{H}_{2} / \mathrm{cm}^{3}$ at a temperature of $11.3 \mathrm{~K}$ and a distance of $140 \mathrm{pc}$, as we find for the mean of the cores in Taurus in this work.

temperature-density relation. Cores extracted from, and characterized using, unfiltered Herschel data typically have higher temperatures and densities than their counterparts in the SCUBA-2 data. This is due to extended emission along the line of sight, which is removed by the filtering process. This further confirmed that spatial filtering was necessary to accurately compare SCUBA-2 and Herschel data.
We found that SCUBA-2 detects only the densest starless cores, with no SCUBA-2 cores having densities below $\sim 6 \times 10^{3} \mathrm{H}_{2} / \mathrm{cm}^{3}$, an order of magnitude higher density than the least dense filtered 250- $\mu \mathrm{m}$-detected Herschel core. There is no equivalent cut-off in temperature, with both SCUBA-2 and Herschel sources having temperatures in the range $\sim 9-20 \mathrm{~K}$. Neither are SCUBA- 2 cores typically smaller in radius than $\mathrm{Herschel}$ cores - i.e. the spatial filtering 
introduced by SCUBA-2 does not appear to change the measured FWHM of a starless core observed at this distance.

Thus, we found that the criterion for whether a starless or prestellar core detected in Herschel data will also be detected in SCUBA-2 data is its density (for a given temperature). In the case of Taurus, for SCUBA-2 GBS Internal Release 1 data, this was $\sim 6 \times 10^{3} \mathrm{H}_{2} / \mathrm{cm}^{3}$. This corresponds to a cut-off in surface brightness, below which SCUBA-2 is no longer sensitive. This makes SCUBA-2 ideal for selecting those cores in Herschel catalogues that are closest to forming stars. This information can be used in the analysis of SCUBA-2 and Herschel data of other GBS regions, as shown in Fig. 10, and to plan future SCUBA-2 observing campaigns.

\section{ACKNOWLEDGEMENTS}

The JCMT has historically been operated by the Joint Astronomy Centre on behalf of the Science and Technology Facilities Council of the United Kingdom, the National Research Council of Canada, and the Netherlands Organization for Scientific Research. Additional funds for the construction of SCUBA-2 were provided by the Canada Foundation for Innovation. Herschel is an ESA space observatory with science instruments provided by European-led Principal Investigator consortia and with important participation from NASA. DWT wishes to thank STFC for FEC support under grant number ST/K002023/1. KP wishes to thank STFC for postdoctoral support under grant numbers ST/K002023/1 and ST/M000877/1 and studentship support under grant number ST/K501943/1.

\section{REFERENCES}

André P., Ward-Thompson D., Barsony M., 1993, ApJ, 406, 122

André P., Ward-Thompson D., Barsony M., 2000, in Mannings V., Boss A. P., Russell S. S., eds, Protostars and Planets IV. University of Arizona Press, Tucson, p. 59

André P. et al., 2010, A\&A, 518, L102

André P., Di Francesco J., Ward-Thompson D., Inutsuka S.-I., Pudritz R. E., Pineda J. E., 2014, From Filamentary Networks to Dense Cores in Molecular Clouds: Toward a New Paradigm for Star Formation. Protostars and Planets VI. University of Arizona Press, Tucson, p. 27

Ballesteros-Paredes J., Klessen R. S., Vázquez-Semadeni E., 2003, ApJ, 592, 188

Barnard E. E., 1907, ApJ, 25, 218

Beckwith S. V. W., Sargent A. I., 1991, ApJ, 381, 250

Beckwith S. V. W., Sargent A. I., Chini R. S., Guesten R., 1990, AJ, 99, 924

Beichman C. A., Myers P. C., Emerson J. P., Harris S., Mathieu R., Benson P. J., Jennings R. E., 1986, ApJ, 307, 337

Benson P. J., Myers P. C., 1989, ApJS, 71, 89

Bintley D. et al., 2014, in Holland W. S., Zmuidzinas J., eds, Society of Photo-Optical Instrumentation Engineers (SPIE) Conference Series, Vol. 9153, Millimeter, Submillimeter, and Far-Infrared Detectors and Instrumentation for Astronomy VII, p. 915303

Bonnor W. B., 1956, MNRAS, 116, 351

Buckle J. V. et al., 2015, MNRAS, 449, 2472

Chapin E. L., Berry D. S., Gibb A. G., Jenness T., Scott D., Tilanus R. P. J., Economou F., Holland W. S., 2013, MNRAS, 430, 2545

Dempsey J. T. et al., 2013, MNRAS, 430, 2534

Di Francesco J., Evans N. J. I., Caselli P., Myers P. C., Shirley Y., Aikawa Y., Tafalla M., 2007, in Reipurth B., Jewitt D., Tucson K. K., eds, Protostars and Planets V, An Observational Perspective of Low-Mass Dense Cores I: Internal Physical and Chemical Properties. University of Arizona Press, Tucson, p. 17

Draine B. T., Lee H. M., 1984, ApJ, 285, 89

Draine B. T. et al., 2007, ApJ, 663, 866

Ebert R., 1955, Z. für Astrophys., 37, 217
Elmegreen B. G., Falgarone E., 1996, ApJ, 471, 816

Friesen R. K., Johnstone D., Naylor D. A., Davis G. R., 2005, MNRAS, 361,460

Griffin M. J. et al., 2010, A\&A, 518, L3

Hacar A., Tafalla M., Kauffmann J., Kovács A., 2013, A\&A, 554, A55

Hildebrand R. H., 1983, QJRAS, 24, 267

Holland W. S. et al., 2013, MNRAS, 430, 2513

Jeans J. H., 1928, Astronomy and Cosmogony. Cambridge Univ. Press, Cambridge

Kirk J. M. et al., 2013, MNRAS, 432, 1424

Lada C., 1987, in Jugaku M. P. J., ed., IAU Symp. 115, Star Formation - From OB Associations to Protostars. D. Reidel Publishing Co, Dordrecht, p. 1

Lee C. W., Myers P. C., Tafalla M., 2001, ApJS, 136, 703

Lynds B. T., 1962, ApJS, 7, 1

Marsh K. A. et al., 2014, MNRAS, 439, 3683

Marsh K. A. et al., 2016, MNRAS, 459, 342

Martin P. G. et al., 2012, ApJ, 751, 28

Men'shchikov A. et al., 2010, A\&A, 518, L103

Men'shchikov A., André P., Didelon P., Motte F., Hennemann M., Schneider N., 2012, A\&A, 542, A81

Molinari S. et al., 2010, A\&A, 518, L100

Nutter D., Stamatellos D., Ward-Thompson D., 2009, MNRAS, 396, 1851

Palmeirim P. et al., 2013, A\&A, 550, A38

Pattle K. et al., 2015, MNRAS, 450, 1094

Pilbratt G. L. et al., 2010, A\&A, 518, L1

Poglitsch A. et al., 2010, A\&A, 518, L2

Sadavoy S. I. et al., 2013, ApJ, 767, 126

Schnee S. et al., 2010, ApJ, 708, 127

Seo Y. M. et al., 2015, ApJ, 805, 185

Shirley Y. L., Nordhaus M. K., Grcevich J. M., Evans,II N. J., Rawlings J. M. C., Tatematsu K., 2005, ApJ, 632, 982

Stamatellos D., Whitworth A. P., Ward-Thompson D., 2007, MNRAS, 379, 1390

Strom S. E., Strom K. M., Grasdalen G. L., 1975, ARA\&A, 13, 187

Swinyard B. M. et al., 2010, A\&A, 518, L4

Ward-Thompson D., Robson E. I., Whittet D. C. B., Gordon M. A., Walther D. M., Duncan W. D., 1989, MNRAS, 241, 119

Ward-Thompson D., Scott P. F., Hills R. E., André P., 1994, MNRAS, 268, 276

Ward-Thompson D., André P., Kirk J. M., 2002, MNRAS, 329, 257

Ward-Thompson D., André P., Crutcher R., Johnstone D., Onishi T., Wilson C., 2007a, in Reipurth B., Jewitt D., Tucson K. K., eds, Protostars and Planets V, University of Arizona Press, Tucson, p. 33

Ward-Thompson D. et al., 2007b, PASP, 119, 855

Ward-Thompson D. et al., 2010, A\&A, 518, L92

Wilking B. A., Lada C. J., Young E. T., 1989, ApJ, 340, 823

Young C. H. et al., 2004, ApJS, 154, 396

\section{SUPPORTING INFORMATION}

Additional Supporting Information may be found in the online version of this article:

Table A1. CSAR output for Herschel sources.

Table A2. CSAR output for filtered-Herschel sources.

Table A3. Derived properties of Herschel sources.

Table A4. Derived properties of filtered-Herschel sources. (http://www.mnras.oxfordjournals.org/lookup/suppl/doi: 10.1093/mnras/stw1978/-/DC1).

Please note: Oxford University Press is not responsible for the content or functionality of any supporting materials supplied by the authors. Any queries (other than missing material) should be directed to the corresponding author for the article. 


\section{APPENDIX A: HERSCHEL SOURCES}

In this Appendix we direct the reader to the full set of sources found in the filtered and unfiltered Herschel $250-\mu \mathrm{m}$ observations of the low-SCUBA-2-variance region of L1495 by CSAR. The unfiltered Herschel sources are shown in Figs A1(a) and (b), while the filtered Herschel sources are shown in Figs A2(a) and (b). For the Herschel sources, at Marsh et al. (2016), Table A1 lists the name, right ascension and declination, measured major and minor FWHM sizes, position angle, flux densities as measured in $\mathrm{Her}$ schel $160-\mu \mathrm{m}, 250-\mu \mathrm{m}, 350-\mu \mathrm{m}$ and $500-\mu \mathrm{m}$ emission, an extrapolated SCUBA-2 850- $\mu \mathrm{m}$ flux density (given in brackets), and the equivalent source in the Marsh et al. (2016) catalogue. Table A2, at the DOI given above, lists the same properties for the filteredHerschel sources as given in Table A1, except the 160- $\mu \mathrm{m}, 250-\mu \mathrm{m}$, $350-\mu \mathrm{m}$ and $500-\mu \mathrm{m}$ flux densities are measured in filtered Herschel emission, and the equivalent source in the Herschel catalogue is listed. The derived source properties, temperature, mass, density and deconvolved FWHM, are listed in Table A3 at the DOI above for the Herschel sources, and in Table A4 at the DOI above for the filtered-Herschel sources.

Many of the sources found in the unfiltered Herschel data do not have counterparts in the Marsh et al. (2016) catalogue. Marsh et al. (2016) identify sources using the getsources algorithm (Men'shchikov et al. 2012), and produce two catalogues: an 'unfiltered' catalogue, and a 'filtered' catalogue of robustly detected cores, inclusion of a core into which requires a stringent set of criteria to be met (Marsh et al. 2016). The Marsh et al. (2016) sources listed in the final column of Table A1 are from their filtered catalogue. We find that, when comparing our sources with the full Marsh et al. (2016) catalogue, approximately half of the Herschel sources without filtered Marsh et al. (2016) counterparts can be identified with a source in the unfiltered Marsh et al. (2016) catalogue (K. Marsh, private communication). The remainder of the sources are typically highly elongated and have a low signal-tonoise ratio, suggesting that with our chosen parameters for CSAR (optimized for detection of sources in the SCUBA-2 data), we may be detecting striations such as those identified by Palmeirim et al. (2013). We therefore caution the reader that this is not the definitive Herschel catalogue for this region. For the definitive Herschel catalogue, please see Marsh et al. (2016).

\section{APPENDIX B: DEMONSTRATION OF SURFACE BRIGHTNESS EFFECT}

In this appendix we demonstrate that the increased number of sources detected in the spatially filtered Herschel $250-\mu \mathrm{m}$ data compared to the number detected in the $850-\mu \mathrm{m}$ SCUBA-2 data is due to the blackbody function peaking at $\sim 250 \mu \mathrm{m}$, while $850 \mu \mathrm{m}$ is on the Rayleigh-Jeans tail, at temperatures typically found in molecular clouds. Hence, dust at temperatures typically found in starless cores emits more flux at Herschel wavelengths than at SCUBA-2 850- $\mu \mathrm{m}$ wavelength, because Herschel wavelengths coincide with the peak of the blackbody function. For the same absolute noise level, any given core will appear at a higher signal-to-noise ratio to Herschel than to SCUBA-2.

To test the hypothesis that this effect causes the increased detection of sources in the filtered Herschel $250-\mu \mathrm{m}$ data over the SCUBA-2 850- $\mu \mathrm{m}$ data, we scaled the noise on the Herschel data such that the relative, rather than the absolute, sensitivity of the Herschel and SCUBA-2 maps became comparable. In order to as closely mimic the SCUBA-2 data as possible, we added noise to
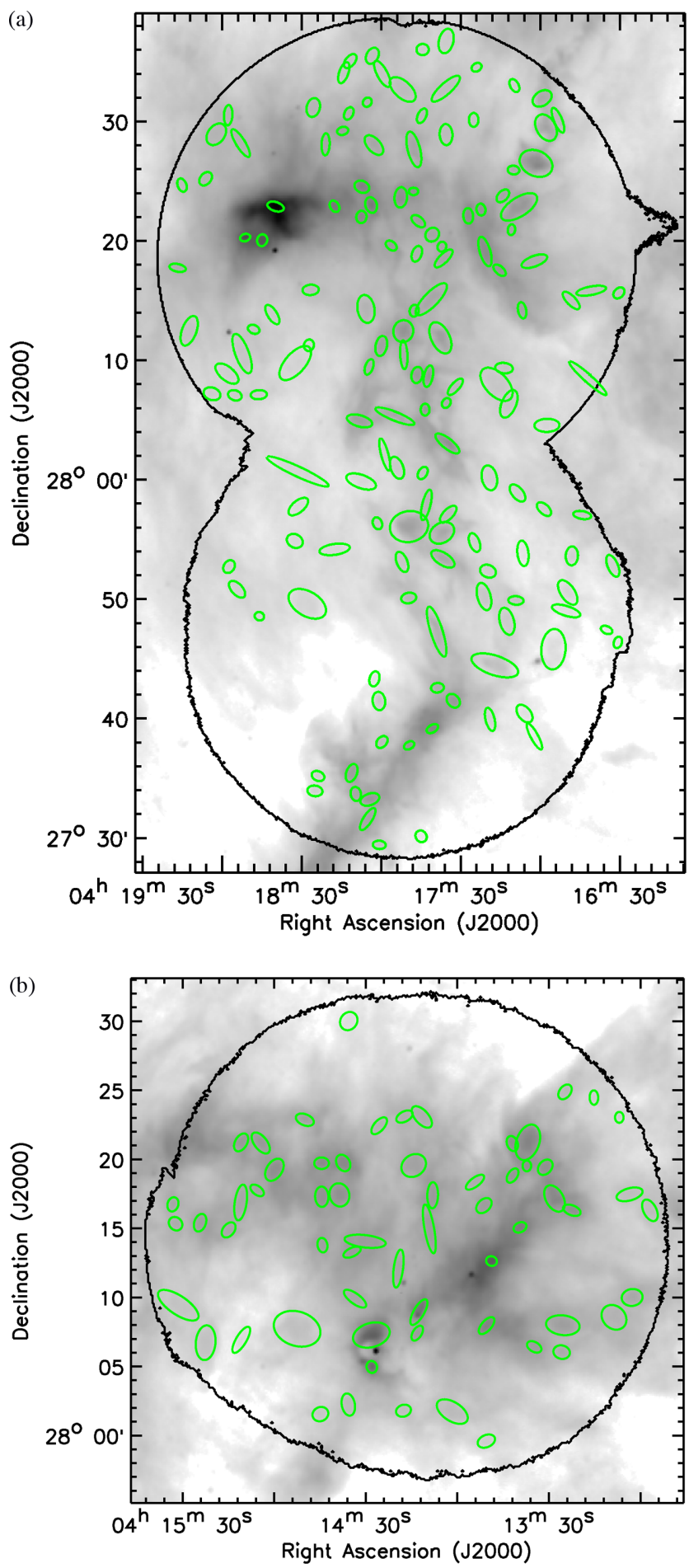

Figure A1. (a) Grey-scale image of the head of the L1495 filament, in Herschel $250 \mu \mathrm{m}$ emission. Sources detected in Herschel $250 \mu \mathrm{m}$ emission are marked by small ellipses. The large-scale contour surrounds the region of lowest SCUBA-2 variance (cf. Buckle et al. 2015). (b) As Fig. A1(a) for the L1495 West region.

the unfiltered Herschel data before passing the data through the SCUBA-2 pipeline.

To do this, we determined the relative brightness of the 250$\mu \mathrm{m}$ and $850-\mu \mathrm{m}$ points on the grey-body curve, for a temperature 
(a)

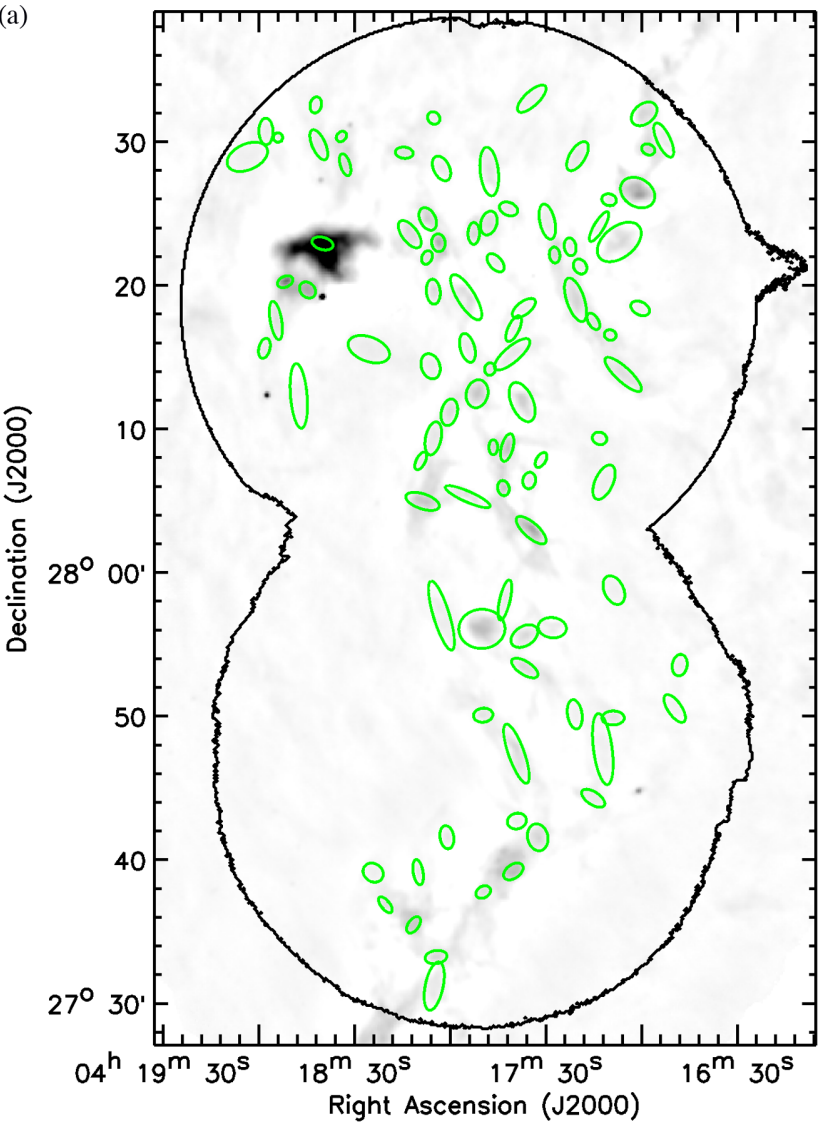

(b)

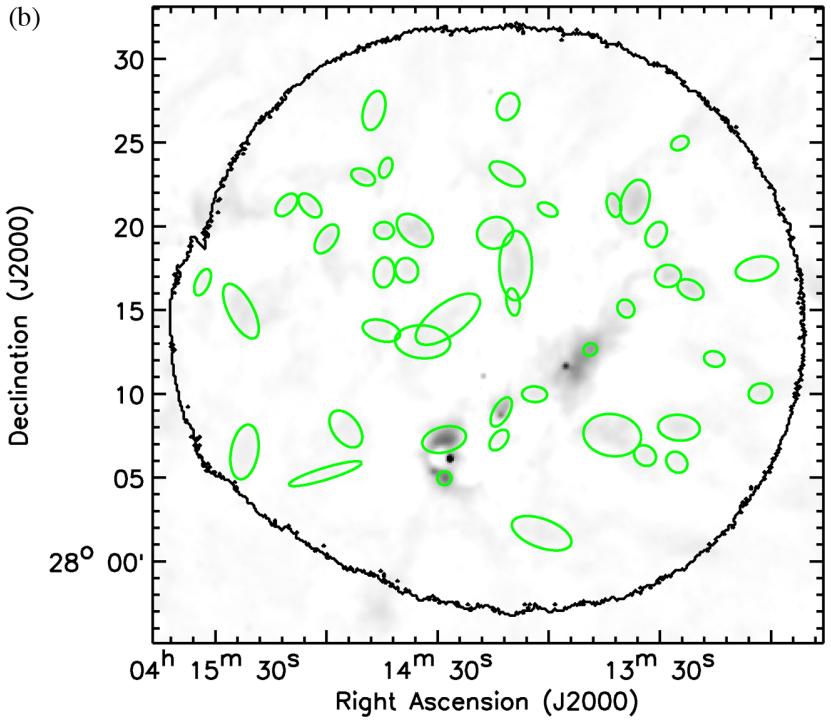

Figure A2. (a) Grey-scale image of the head of the L1495 filament, in filtered Herschel $250 \mu \mathrm{m}$ emission. Sources detected in filtered Herschel $250 \mu \mathrm{m}$ emission are marked by small ellipses. The large-scale contour surrounds the region of lowest SCUBA-2 variance (cf. Buckle et al. 2015). (b) As Fig. A2(a), for the L1495 West region.
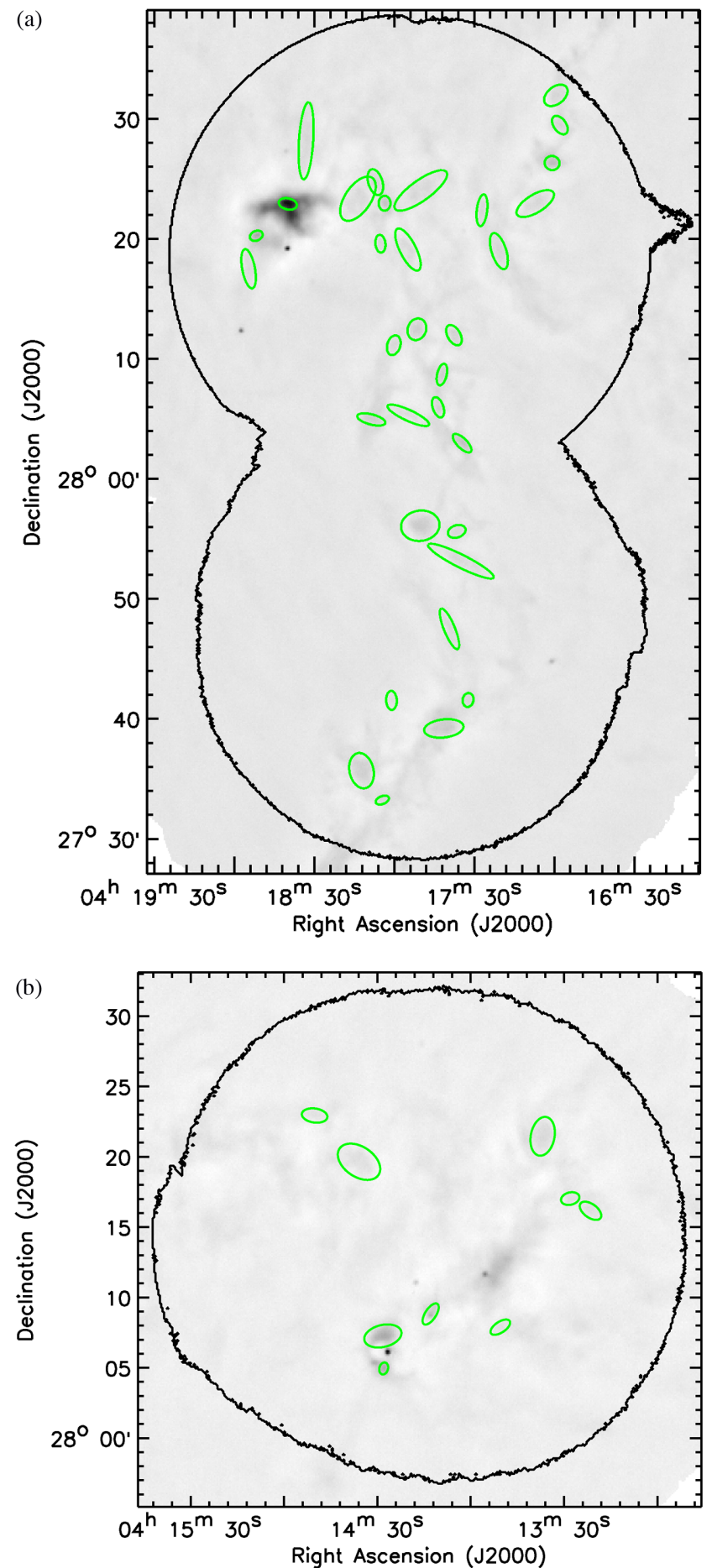

Figure B1. (a) Grey-scale image of the head of the L1495 filament, in increased-noise filtered Herschel $250 \mu \mathrm{m}$ emission. Sources detected in increased-noise filtered Herschel $250 \mu \mathrm{m}$ emission are marked by small ellipses. The large-scale contour surrounds the region of lowest SCUBA-2 variance (cf. Buckle et al. 2015). This should be compared with Fig. 4(a). (b) As Fig. B1(a), for the L1495 West region. This should be compared with Fig. 4(b). 


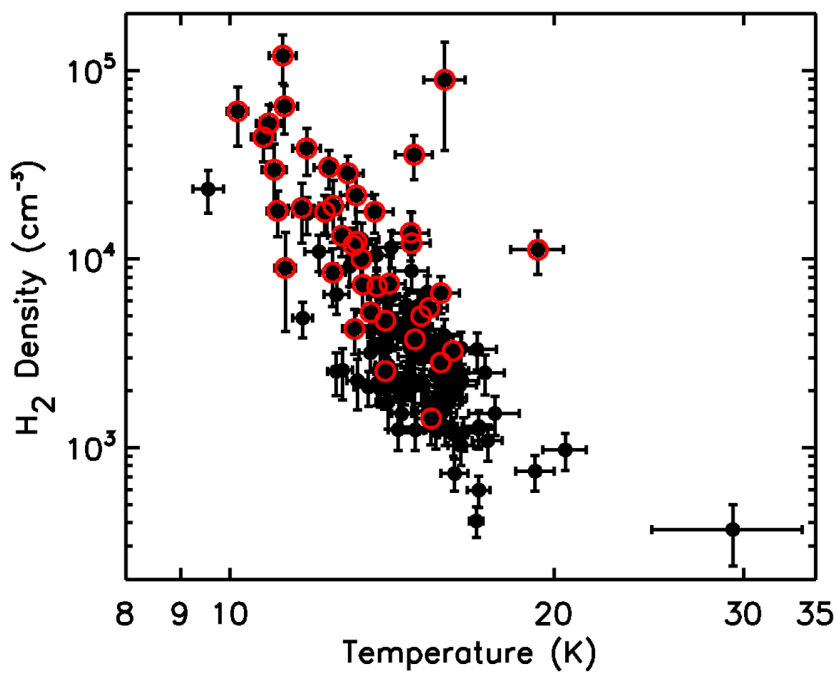

Figure B2. A comparison of the temperature and density of the filteredHerschel $250-\mu \mathrm{m}$ sources, with those sources which are detected in the increased-noise filtered Herschel data marked with a red circle. Note the tendency for the sources detected in the increased-noise data to be among the denser sources.

$T=11.3 \mathrm{~K}$ (the mean temperature of our cores), and for a dust opacity index $\beta=1.3$ (as we use in the analysis in this work), i.e.

$\frac{F_{250}}{F_{850}}=\left(\frac{\nu_{250}}{v_{850}}\right)^{3+\beta} \frac{\mathrm{e}^{\frac{h \nu_{850}}{\mathrm{~B}^{T}}}-1}{\mathrm{e}^{\frac{h \nu_{250}}{\mathrm{~B}^{T}}}-1}=4.11$.

We measured the rms noise level of the SCUBA-2 850- $\mu$ m map to be $0.9 \mathrm{mJy} / 6$-arcsec pixel. Hence, our target rms value for the Herschel data was $4.11 \times 0.9 \mathrm{mJy} / 6$-arcsec pixel, i.e. $3.7 \mathrm{mJy} / 6$ arcsec pixel. We determined the rms noise level that needed to be added to the original map by subtracting the measured rms noise level from the target noise level in quadrature. We created a Gaussian noise distribution of the required amplitude, and added this to the unfiltered Herschel 250- $\mu \mathrm{m}$ map.

We then passed the increased-noise Herschel 250- $\mu \mathrm{m}$ map through the SCUBA-2 pipeline in the manner described in Section 4, removing the large-scale structure from the Herschel data.

We extracted sources from our increased-noise filtered Herschel 250- $\mu \mathrm{m}$ map with CSAR, using the parameters described in Section 4 . We extracted 47 sources from the region of lowest SCUBA-2 variance, of which five were excluded as being point sources. This left 42 detections of sources likely to be starless cores. We detected 25 starless cores in the $850-\mu \mathrm{m}$ SCUBA- 2 data. While we detect more cores in the increased-noise filtered Herschel 250- $\mu \mathrm{m}$ map, the cores we identify are similar to those detected in SCUBA-2 data: they are located along the dense filaments, and many can be directly associated with sources in the SCUBA- 2 catalogue. Particularly, the low-surface-brightness sources away from the dense filaments are no longer detected, as expected. The starless cores which we detect in the increased-noise filtered Herschel 250- $\mu \mathrm{m}$ map are listed in Table B1 and shown in Figs B1(a) and (b).

In Fig. B2 we plot the temperatures and densities of the filteredHerschel 250- $\mu \mathrm{m}$ sources as determined in Section 4 (cf. Fig. 8). Those filtered-Herschel sources which are detected in the increasednoise filtered Herschel data (i.e. those sources which are listed as counterpart sources in Table B1) are highlighted. It can be seen that those sources detected in the increased-noise filtered Herschel data are preferentially the denser sources. The mean density of the
Table B1. Sources detected in increased-noise filtered Herschel 250- $\mu \mathrm{m}$ data.

\begin{tabular}{|c|c|c|c|c|c|}
\hline $\begin{array}{l}\text { Source } \\
\text { Index }\end{array}$ & $\begin{array}{l}\text { R.A. } \\
(\mathrm{J} 2000)\end{array}$ & $\begin{array}{l}\text { Dec. } \\
(\mathrm{J} 2000)\end{array}$ & $\begin{array}{l}\text { FWHM } \\
(\operatorname{arcsec})\end{array}$ & $\begin{array}{c}\text { Angle } \\
\left({ }^{\circ} \mathrm{E} \text { of } \mathrm{N}\right)\end{array}$ & $\begin{array}{c}\text { Counterpart } \\
\text { Sources }\end{array}$ \\
\hline N1 & $4: 18: 40.53$ & $+28: 23: 02.2$ & $7.7 \times 4.6$ & 77.4 & $\mathrm{~S} 1, \mathrm{~F} 1$ \\
\hline N2 & $4: 14: 27.71$ & $+28: 07: 12.8$ & $13.5 \times 7.8$ & 103.5 & $\mathrm{~S} 10, \mathrm{~F} 3$ \\
\hline N3 & $4: 18: 52.44$ & $+28: 20: 22.1$ & $5.5 \times 4.0$ & 113.0 & $\mathrm{~S} 23, \mathrm{~F} 2$ \\
\hline N4 & $4: 14: 27.50$ & $+28: 04: 53.9$ & $4.3 \times 3.1$ & 164.8 & $\mathrm{~S} 13, \mathrm{~F} 4$ \\
\hline N5 & $4: 14: 12.23$ & $+28: 08: 45.4$ & $8.6 \times 3.9$ & 146.8 & S15,F5 \\
\hline N6 & 4:17:00.29 & $+28: 26: 28.2$ & $6.2 \times 5.9$ & 80.0 & S8,F6 \\
\hline N7 & $4: 17: 34.40$ & $+28: 03: 05.5$ & $10.2 \times 4.1$ & 45.3 & $\mathrm{~S} 12, \mathrm{~F} 8$ \\
\hline N8 & $4: 18: 03.83$ & $+28: 23: 06.4$ & $6.2 \times 4.9$ & 179.9 & S6,F10 \\
\hline N9 & $4: 17: 50.17$ & $+27: 56: 11.8$ & $16.1 \times 12.6$ & 97.6 & S3,F12 \\
\hline N10 & $4: 17: 42.04$ & $+28: 08: 48.8$ & $9.2 \times 4.0$ & 166.8 & $\mathrm{~S} 2, \mathrm{~F} 13$ \\
\hline N11 & $4: 17: 43.49$ & $+28: 06: 05.0$ & $8.8 \times 4.4$ & 18.7 & S7,F15 \\
\hline N12 & $4: 17: 41.24$ & $+27: 39: 17.2$ & $16.5 \times 7.5$ & 96.6 & $\mathrm{~S} 18, \mathrm{~F} 11$ \\
\hline N13 & $4: 18: 12.20$ & $+27: 35: 45.1$ & $14.9 \times 10.0$ & 12.8 & S9,F17 \\
\hline N14 & 4:18:08.72 & $+28: 05: 03.8$ & $12.1 \times 4.3$ & 75.5 & S5,F14 \\
\hline N15 & $4: 17: 51.53$ & $+28: 12: 35.5$ & $9.2 \times 7.6$ & 158.3 & S14,F16 \\
\hline N16 & $4: 17: 37.51$ & $+28: 12: 06.2$ & $9.3 \times 5.3$ & 29.5 & S21,F19 \\
\hline N17 & $4: 18: 07.34$ & $+28: 24: 51.8$ & $11.1 \times 5.9$ & 17.0 & $\mathrm{~S} 24, \mathrm{~F} 18$ \\
\hline N18 & $4: 16: 57.33$ & $+28: 29: 38.3$ & $8.7 \times 5.2$ & 35.4 & $\mathrm{~S} 17, \mathrm{~F} 20$ \\
\hline N19 & 4:18:04.37 & $+27: 33: 18.0$ & $5.8 \times 3.3$ & 111.7 & F25 \\
\hline N20 & $4: 17: 39.12$ & $+27: 47: 35.5$ & $18.2 \times 4.5$ & 22.3 & F26 \\
\hline N21 & $4: 16: 58.84$ & $+28: 32: 06.9$ & $11.2 \times 7.1$ & 128.7 & F27 \\
\hline N22 & $4: 18: 33.65$ & $+28: 28: 18.3$ & $32.2 \times 5.9$ & 176.3 & F29 \\
\hline $\mathrm{N} 23$ & $4: 13: 35.62$ & $+28: 21: 21.9$ & $14.0 \times 8.4$ & 169.1 & F21 \\
\hline N24 & $4: 17: 26.89$ & $+28: 22: 31.8$ & $13.3 \times 4.3$ & 173.4 & F28 \\
\hline N25 & 4:18:05.39 & $+28: 19: 43.5$ & $7.5 \times 4.2$ & 6.2 & F37 \\
\hline N26 & $4: 18: 00.27$ & $+28: 11: 15.6$ & $8.4 \times 5.4$ & 163.5 & S19,F39 \\
\hline N27 & $4: 17: 20.48$ & $+28: 19: 07.2$ & $15.7 \times 6.1$ & 17.7 & $\mathrm{~S} 25, \mathrm{~F} 24$ \\
\hline N28 & $4: 18: 55.31$ & $+28: 17: 36.8$ & $16.7 \times 5.3$ & 10.8 & F44 \\
\hline N29 & $4: 17: 32.10$ & $+27: 41: 39.7$ & $5.8 \times 4.5$ & 170.1 & F22 \\
\hline N30 & $4: 13: 26.87$ & $+28: 16: 54.6$ & $6.7 \times 4.4$ & 104.1 & F34 \\
\hline N31 & 4:14:35.08 & $+28: 19: 38.3$ & $16.7 \times 10.7$ & 55.8 & F45 \\
\hline N32 & $4: 17: 34.83$ & $+27: 53: 14.9$ & $30.6 \times 5.4$ & 63.3 & F35 \\
\hline N33 & 4:17:06.70 & $+28: 23: 05.1$ & $17.7 \times 7.5$ & 121.5 & F33 \\
\hline N34 & $4: 17: 50.11$ & $+28: 24: 10.5$ & $26.4 \times 8.5$ & 125.8 & F40 \\
\hline N35 & $4: 17: 36.40$ & $+27: 55: 43.6$ & $7.6 \times 5.1$ & 108.4 & F36 \\
\hline N36 & $4: 13: 20.35$ & $+28: 16: 01.7$ & $8.8 \times 4.8$ & 53.8 & F41 \\
\hline N37 & $4: 18: 00.96$ & $+27: 41: 37.3$ & $7.9 \times 4.4$ & 2.9 & F72 \\
\hline N38 & $4: 13: 49.76$ & $+28: 07: 47.1$ & $8.0 \times 3.8$ & 123.5 & None \\
\hline N39 & $4: 17: 54.95$ & $+28: 19: 14.6$ & $19.4 \times 6.7$ & 26.9 & F43 \\
\hline N40 & $4: 18: 13.93$ & $+28: 23: 28.7$ & $21.1 \times 10.8$ & 145.8 & F38 \\
\hline N41 & $4: 17: 54.74$ & $+28: 05: 24.7$ & $19.0 \times 4.5$ & 65.3 & F53 \\
\hline N42 & $4: 14: 49.36$ & $+28: 22: 57.1$ & $9.1 \times 5.0$ & 82.0 & F49 \\
\hline
\end{tabular}

filtered-Herschel sources is $9.0 \times 10^{3} \mathrm{H}_{2} \mathrm{~cm}^{-3}$, while the median density is $3.5 \times 10^{3} \mathrm{H}_{2} / \mathrm{cm}^{3}$. The mean density of the subset of the filtered-Herschel sources which are detected in the increasednoise data is $21.4 \times 10^{3} \mathrm{H}_{2} / \mathrm{cm}^{3}$, approximately 2.4 times that of the set as a whole, while the median density of the subset detected in the increased-noise data is $12.1 \times 10^{3} \mathrm{H}_{2} / \mathrm{cm}^{3}$, approximately 3.5 times that of the whole data set. Moreover, 77 per cent of the filtered-Herschel sources with densities $\geq 10^{4} \mathrm{H}_{2} / \mathrm{cm}^{3}$ are detected in the increased-noise data, while only 16 per cent of the filteredHerschel sources with densities $<10^{4} \mathrm{H}_{2} / \mathrm{cm}^{3}$ are detected.

The clear cut-off in density which is apparent in the detection of SCUBA-2 850- $\mu \mathrm{m}$ sources is less distinct in the increased-noise filtered-Herschel data. This could be due to variation in temperature of the sources, meaning that our choice of increasing the rms noise to a factor of 4.11 times the SCUBA-2 $850-\mu \mathrm{m}$ noise is not representative of all sources. Resolution effects may also contribute to this. However, these results support our hypothesis that the tendency 
for SCUBA-2 850- $\mu \mathrm{m}$ emission to detect the only densest sources is due to the decreased surface brightness of sources at $850-\mu \mathrm{m}$ relative to $250-\mu \mathrm{m}$.

${ }^{1}$ Jeremiah Horrocks Institute, University of Central Lancashire, Preston PRI 2HE, UK

${ }^{2}$ School of Physics \& Astronomy, Cardiff University, The Parade, Cardiff CF24 3AA, UK

${ }^{3}$ Astrophysics Group, Cavendish Laboratory, J J Thomson Avenue, Cambridge CB3 OHE, UK

${ }^{4}$ Kavli Institute for Cosmology, Institute of Astronomy, University of Cambridge, Madingley Road, Cambridge CB3 OHA, UK

${ }^{5}$ Department of Physics and Astronomy, University of Exeter, Stocker Road, Exeter EX4 4QL, UK

${ }^{6}$ NRC Herzberg Astronomy and Astrophysics, 5071 West Saanich Rd, Victoria, BC V9E 2E7, Canada

${ }^{7}$ Department of Physics and Astronomy, University of Victoria, Victoria, $B C$ V8P 1A1, Canada

${ }^{8}$ Laboratoire AIM CEA/DSM-CNRS-Université Paris Diderot, IRFU/Service d'Astrophysique, CEA Saclay, F-91191 Gif-sur-Yvette, France
${ }^{9}$ Department of Physics and Astronomy, University of Waterloo, Waterloo, Ontario N2L 3G1, Canada

${ }^{10}$ East Asian Observatory, 660 N. A'ohōkū Place, University Park, Hilo, Hawaii 96720, USA

${ }^{11}$ LSST Project Office, 933 N. Cherry Ave, Tucson, AZ 85719, USA

${ }^{12}$ Leiden Observatory, Leiden University, PO Box 9513, 2300RA Leiden, the Netherlands

${ }^{13}$ Max-Planck Institute for Astronomy, Königstuhl 17, D-69117 Heidelberg, Germany

${ }^{14}$ Max-Planck-Institut für extraterrestrische Physik, Giessenbachstrasse 1, D-85748 Garching, Germany

${ }^{15}$ Department of Physical Sciences, The Open University, Milton Keynes MK7 6AA, UK

${ }^{16}$ The Rutherford Appleton Laboratory, Chilton, Didcot OX11 ONL, UK

${ }^{17}$ Joint ALMA Observatory, Alonso de Córdova 3107, Vitacura - Santiago, Chile

${ }^{18}$ Istituto di Astrofisica e Planetologia Spaziali-INAF, Via Fosso del Cavaliere 100, I-00133 Roma, Italy

This paper has been typeset from a $\mathrm{T}_{\mathrm{E}} \mathrm{X} / \mathrm{L} \mathrm{T} \mathrm{E} \mathrm{X}$ file prepared by the author. 